\title{
The attachment of colloidal particles to environmentally relevant surfaces and the effect of particle shape.
}

\author{
Coy P. McNew ${ }^{\mathrm{a}, \mathrm{b}}$, Negin Kananizadeh ${ }^{\mathrm{c}}$, Yusong Li ${ }^{\mathrm{c}}$, Eugene J. LeBoeuf ${ }^{\mathrm{b}, *}$ \\ ${ }^{a}$ Land, Air, and Water Resources, University of California Davis, Davis, California \\ 95616, USA \\ ${ }^{b}$ Department of Civil and Environmental Engineering, Vanderbilt University, Nashville, \\ Tennessee 37235, USA \\ ${ }^{c}$ Department of Civil Engineering, University of Nebraska-Lincoln, Lincoln, Nebraska \\ 68588, USA
}

\section{Abstract}

Despite the prevalence of nonspherical colloidal particles, the role of particle shape in the transport of colloids is largely understudied. This study investigates the attachment of colloidal particles onto environmentally relevant surfaces while varying particle shape and ionic strength. Using quartz crystal microbalance and atomic force microscopy measurements, the role of particle shape was elucidated and possible mechanisms discussed. The attachment of both spherical and stretched polystyrene colloidal particles onto a smooth alginate-coated silica surface showed qualitative agreement with DLVO theory. Attachment onto a Harpeth humic acid (HHA) surface, however, significantly deviated from DLVO theory due to it's high surface heterogeneity and extended confirmation from the silica surface. This extended confirmation provided increased potential for spherical particle entanglement, while the enlarged major axis of the stretched particles hindered their ability to

\footnotetext{
*Corresponding author

Email address: eugene.j.leboeuf@vanderbilt.edu (Eugene J. LeBoeuf)
} 
attach. As ionic strength increased, the HHA layer condensed and provided less potential for spherical particle entanglement and therefore the selectivity for spherical particle attachment vanished. The findings presented in this study suggest that colloidal particle shape may play a complex and important role in predicting the transport of colloidal particles, especially in the presence of natural organic matter-coated surfaces.

Keywords: QCM-D, transport, colloid shape, attachment, NOM

\section{Introduction}

Colloidal transport through the subsurface environment is a topic of 3 great importance and intense study to the field of environmental engineering.

4 Seemingly immobile toxins can attach to the surface of mobile colloids and be 5 readily transported through the subsurface, greatly enhancing their risk to 6 ecosystems and human health, or in some cases, the colloid itself is the toxin 7 of concern (Ward et al., 1985). Many natural colloids are nonspherical in 8 shape, including plate and rod shaped clay (Degueldre et al., 1996), various 9 bacterial shapes (e.g. ellipsoid, ovoid, and rod shaped) (Hirsch and RadesRohkohl, 1990; Balkwill et al., 1989), and some engineered nanomaterials 11 (e.g. carbon nanotubes) which exhibit an aquatic toxic potential (Nel et al. 2006). Despite the ubiquity of nonspherical colloids, only a limited number of studies have focused on the role of shape with regard to colloidal transport (Seymour et al., 2013; Baltus et al., 2008; Wang et al., 2008; Salerno et al., 2006; Weiss et al., 1995).

Weiss et al. (1995) demonstrated the systematic effect of bacterial cell shape on the transport of bacterial cells in packed columns and Salerno et al. 
(2006) observed higher retention of polystyrene latex microspheres with increasing aspect ratio, illustrating the direct impact of particle shape on retention in porous media. Furthermore, two additional studies Baltus et al. (2008); Wang et al. (2008) examined the microfiltration of various types of bacterial cells, also demonstrating a sensitivity to cell shape, with Wang et al. (2008) suggesting that cell shape may be the determining factor in the filterability of bacteria. Aside from advection, dispersion, and diffusion, colloidal transport also depends on the kinetic rate at which particles attach to soil and grain surfaces with which they collide. To date, only one study has attempted to isolate the role of shape on particle deposition. Seymour et al. (2013) reported a much higher deposition rate of spherical carboxylatemodified polystyrene particles as compared to 2:1 and 4:1 stretched versions of the same particles. These experiments, however, were conducted under favorable conditions (i.e. a positively charged, PLL-coated silica surface), lacking an energy barrier to deposition and limiting the environmental relevance of the findings. No study to date has investigated the role of shape on colloidal particle attachment to environmentally relevant surfaces.

When dissolved in solution, NOM adsorbs to the surface of colloidal particles and typically limits their deposition through steric hindrance. When the depositional surfaces are pre-coated with NOM, however, the results tend to be much more complex. Pre-coating silica surfaces with various NOM have been shown to hinder, enhance, or have no effect on attachment, depending on the type of NOM (McNew and LeBoeuf, 2015; Chen and Elimelech, 2008), ionic strength of solution (Chang and Bouchard, 2013; Qu et al., 2012; Chen and Elimelech, 2008), and temperature (McNew and LeBoeuf, 2015). 
Chen and Elimelech (2008) reported that an alginate layer enhanced $\mathrm{nC}_{60}$ attachment in the presence of $10 \mathrm{mM} \mathrm{NaCl}$, while hindering it in the presence of $30 \mathrm{mM} \mathrm{NaCl}$. The variable nature was attributed to the alginate layer's compaction at higher $\mathrm{NaCl}$ concentrations, resulting in a smoother, more rigid layer, with less room for $\mathrm{nC}_{60}$ particle entanglement. In our recent work (McNew and LeBoeuf, 2015), we reported that increasing the temperature of a humic acid layer while holding all other variables constant, raised the attachment efficiency of $\mathrm{nC}_{60}$ from low attachment $(\alpha=0.3)$ to complete attachment $(\alpha=1)$. We attributed this to the temperature-dependent hydration of the layer, opening up previously unavailable sorption sites.

․ Derjaguin-Landau-Verwey-Overbeek (DLVO) theory (Derjaguin, 1941; Verwey, 1947) describes the attractive and repulsive forces between a colloidal particle and a surface. Several studies have investigated the attachment of colloidal particles to bare mineral surfaces in simple electrolyte solutions Chowdhury et al. 2014a; Chang and Bouchard, 2013; Shen et al. 2011; Yi and Chen, 2011; Jiang et al., 2010, Chen and Elimelech, 2008, 2006) and the results appear to be in good qualitative agreement with DLVO theory. The attachment deviates significantly from DLVO theory, however, in the presence of natural organic matter (NOM), whether dissolved in solution Akaighe et al., 2013, Qu et al., 2012; Thio et al., 2011; Jiang et al., 2010, Chen and Elimelech, 2008) or coated onto the depositional surface (McNew and LeBoeuf, 2015; Chowdhury et al., 2014b; Chang and Bouchard, 2013; Thio et al., 2011; Qu et al., 2012; Jiang et al., 2010; Tong et al., 2010; Chen and Elimelech, 2008).

The purpose of this study is to isolate the role of shape on the attachment 
of colloidal particles to environmentally relevant surfaces by combining quartz crystal microbalance with dissipation monitoring (QCM-D) and atomic force microscopy (AFM) experiments. For the first time, we present attachment efficiency curves for colloidal particles onto NOM coated silica surfaces, as a function of particle shape and ionic strength. By comparing the attachment results with DLS surface measurements and AFM topography images, we elucidate and discuss the mechanisms driving the complex attachment behavior observed. The results presented here suggest that colloidal particle shape will play an important role in predicting transport, especially in the presence of NOM. A better understanding of the role of shape in the attachment of colloidal particles to environmentally relevant surfaces will lead to a more accurate prediction of colloidal particle transport, aiding in risk assessment of potential exposure pathways.

\section{Materials and Methods}

\subsection{Materials}

$200 \mathrm{~nm}$ carboxylate-modified polystyrene (PS) spheres were purchased from Phosphorex (107, Hopkinton, MA). Dry Harpeth humic acid (HHA) was isolated and characterized as described in our previous publication (DeLapp et al. 2005). Alginic acid sodium salt (alginate, A2158), poly-L-lysine hydrobromide (PLL, P1274), and 4-(2-Hydroxyethyl)-1-piperazineethanesulfonic acid (HEPES) were purchased from Sigma-Aldrich (St. Louis, MO). Reagent grade $\mathrm{NaCl}$ (S671) was purchased from Fisher Scientific (Pittsburgh, PA). Silica-coated QCM-D sensors (QSX-303) were purchased from Q-Sense (Biolin Scientific, Stockholm, Sweden). 900 nm silica microspheres were pur- 
chased from Polysciences Inc (Warrington, PA) as a surrogate for estimating the electrophoretic mobility of the silica sensor surface. All solutions were prepared using ultra pure water purified by the Milli-Q Water Purification System from Millipore (Billerica, MA).

\subsection{Preparation of Stretched PS Particles}

The particle stretching procedure used in this study is based on a method previously published by Champion et al. (2007) and the details can be found in Appendix A.1. In order to determine the concentration of the stretched particles, a calibration curve was constructed using known concentrations of stock PS particles and UV-vis spectrophotomemtry (NanoDrop 2000 Thermo Scientific, Wilmington, DE). The resulting calibration curve can be seen in Appendix A.1. The stock stretched PS suspension was determined to have a concentration of $129 \mathrm{mg} \mathrm{L}^{-1}$.

\subsection{Preparation of HHA Solution}

In order to prepare the HHA solution, $50 \mathrm{mg}$ dry HHA was introduced into $400 \mathrm{~mL}$ deionized water and stirred for more than 3 hours. During this time, the $\mathrm{pH}$ was adjusted drop wise with $0.1 \mathrm{~N} \mathrm{NaOH}$ to a $\mathrm{pH}$ of 10.0 to allow the HHA to dissolve. After nearly complete dissolution of the HHA, the pH was adjusted drop wise with $\mathrm{HCl}$ down to 7.0. The alginate stock solution was prepared by stirring $50 \mathrm{mg}$ dry alginate in $400 \mathrm{~mL}$ deionized water overnight, after which time the alginate was adjusted drop wise with $0.1 \mathrm{~N} \mathrm{NaOH}$ to a $\mathrm{pH}$ of 7.0. Both HHA and alginate stock solutions were filtered through 0.22 $\mu \mathrm{m}$ pore size membrane filter paper (Fisher, GSWP02500) and kept in the dark for the entirety of the study, during which time they remained stable 
to aggregation as confirmed by periodic DLS measurements. The resulting HHA and alginate stock solutions were determined to have a total carbon content (Shimadzu TOC-V CPH/CPN, Columbia, MD) of 110 and $102 \mathrm{mg}$ $\mathrm{L}^{-1}$, respectively.

All prepared electrolyte solutions were filtered through a $0.22 \mu \mathrm{m}$ pore size membrane filter paper and $\mathrm{pH}$ adjusted so that all attachment experiments could take place at a $\mathrm{pH}$ of 7.0 .

\subsection{Characterization Measurements}

Because particle size can control diffusion rate, DLS (Malvern Zetasizer Nano ZS, Worcestershire, UK) was utilized to measure the size of spherical PS particles at each experimental condition investigated. Five independent runs at 1 minute each were performed for each particle size measurement, with each resulting data point consisting of 5 independent measurements.

Image J software was used to measure the length of major and secondary axes of the stretched PS particles. Figs. B.13, B.14, and B.15 show the distribution of major and minor axis of the stretched PS particles and their ratio based on the Image J measurement of 100 particles. Additional shape related parameters including averaged ellipticity, effective diameter, and surface area and the method for calculating these values are provided in Appendix B.

Electrophoretic mobility (EPM) measurements were conducted for the spherical and stretched PS particles at each experimental condition by DLS in conjunction with a universal dip cell (Malvern ZEN1002, Worcestershire, UK). Each reported value of EPM was the result of 10 independent measurements. In order to estimate the EPM values of the silica depositional surfaces utilized in this study, $900 \mathrm{~nm}$ silica microspheres were used as a surrogate. 
EPM values were measured for uncoated, HHA-coated, and alginate-coated silica at each experimental condition. In order to coat the silica microspheres, they were first introduced into a $100 \mathrm{mg} \mathrm{L}^{-1}$ solution of PLL, stirred for 20 minutes and then drained through $0.45 \mu \mathrm{m}$ pore size membrane filter paper (Fisher, GSWP02500) while being rinsed with DI water, allowing all unbound PLL to pass through, but leaving the positively charged, PLL-coated silica particles behind. The process was then repeated for a HHA or alginate coating. All depositional surface EPM measurements were conducted at a silica concentration of $10 \mathrm{mg} \mathrm{L}^{-1}$. Zeta potentials were estimated using the Smoluchowski approximation (Hunter, 2013).

\subsection{Attachment Experiments}

The deposition rate of spherical or stretched PS was monitored using a QCM-D (Q-Sense E4 unit, Biolin Scientific, Stockholm, Sweden) under varying experimental conditions. Flowrates were held constant at $0.1 \mathrm{~mL} \mathrm{~min}^{-1}$, resulting in laminar flow within the module as confirmed by a Reynolds Number of 0.26 Appendix C). PS particle concentration and $\mathrm{pH}$ was held constant at $6 \mathrm{mg} \mathrm{L}^{-1}$ and 7 , respectively, in order to simulate environmentally relevant conditions. All experiments took place at a temperature of 20 ${ }^{\circ} \mathrm{C}$, which was held constant by a Peltier element. Before each experiment, the silica QCM-D sensors were soaked in a 2\% Hellmanex II solution (Mullheim, Germany) overnight, rinsed in DI water, and dried in ultrapure $\mathrm{N}_{2}$. The sensors were then cleaned in a UV-Ozone cleaner (Jelight 42, Irvine, CA) for 20 minutes immediately before being loaded into the QCM-D.

A representative QCM-D experiment and detailed experimental protocol are provided in Appendix A.2. As particles attach to the sensor surface, mass 
increases, corresponding to a continuous decrease in frequency, as described in the Sauerbrey relation (Sauerbrey, 1959). The Sauerbrey relation can be seen in Eq. 1, where $m$ is the mass deposited, $C$ is the crystal constant (17.7 $\mathrm{ng} \mathrm{cm} \mathrm{cm}^{-2} \mathrm{~Hz}^{-1}$ ) and $f_{n}$ is the frequency at overtone $n$.

$$
\Delta m=-C \frac{1}{n} \Delta f_{n}
$$

In order to compare the particle attachment between varying ionic strengths independent of aggregation and diffusion effects, the deposition attachment efficiency $(\alpha)$ was calculated from Eq. 2, where $d f_{3} / d t$ is the initial deposition rate of the third overtone of frequency calculated from the first 120 seconds of frequency drop and $\left(d f_{3} / d t\right)_{\text {diff limited }}$ is the diffusion limited deposition rate, under identical experimental conditions. The diffusion limited deposition rate was measured by flowing the PS particles over the PLL layer at each experimental condition investigated. Since the PS particles are negatively charged and the PLL layer is positively charged, there is no energy barrier to deposition and any PS particles that flow near the PLL surface will attach, therefore the deposition is limited only by diffusion of the PS particles to the sensor surface.

$$
\alpha=\frac{d f_{3} / d t}{\left(d f_{3} / d t\right)_{\text {diff limited }}}
$$

\subsection{AFM Images}

A Bruker Dimension Icon AFM (Billerica, MA) was used to image the HHA and alginate surfaces. The HHA or alginate layer was constructed as described in the experimental procedure, carefully removed from the QCM-D, 
dried in $\mathrm{N}_{2}$, and loaded into the AFM for imaging. The AFM was operated in tapping mode, using a silicon cantilever. The nominal tip characteristics were $8 \mathrm{~nm}$ radius of curvature, $40 \mathrm{~N} \mathrm{~m}^{-1}$ spring constant, and $300 \mathrm{kHz}$ resonant frequency.

\subsection{DLVO Calculations}

The interaction energy between the PS particles and the collector surface was calculated using the surface element integration (SEI) method. SEI is a method for evaluating DLVO interaction energy between a particle and a flat plate (Bhattacharjee and Elimelech, 1997). Unlike the sphere-plate DLVO estimation methods more commonly applied to nanoparticle-plate interactions (Elimelech et al., 2013, Hogg et al., 1966), the SEI method accounts for the orientation dependence of the interaction energy between a flat surface and a spheroidal particle (Bhattacharjee and Elimelech, 1997; Wu et al., 2013). Details of the SEI method are provided in Appendix D.

\section{Results and Discussion}

\subsection{Particle and Surface Characterization}

Spherical and stretched PS particles were investigated by SEM (Hitachi S4700) and representative images can be seen in Fig. $1 \mathrm{a}$ and $1 \mathrm{~b}$, respectively. SEM images of the stretched PS particles were used to quantify the shape of the particles using Image $\mathrm{J}$ software. Major axis diameter, minor axis diameter, ratio of axis diameters, ellipticity, surface area, and effective diameter were all estimated for both the spherical and stretched PS particles. A summary of the shape quantification along with a detailed explanation of 


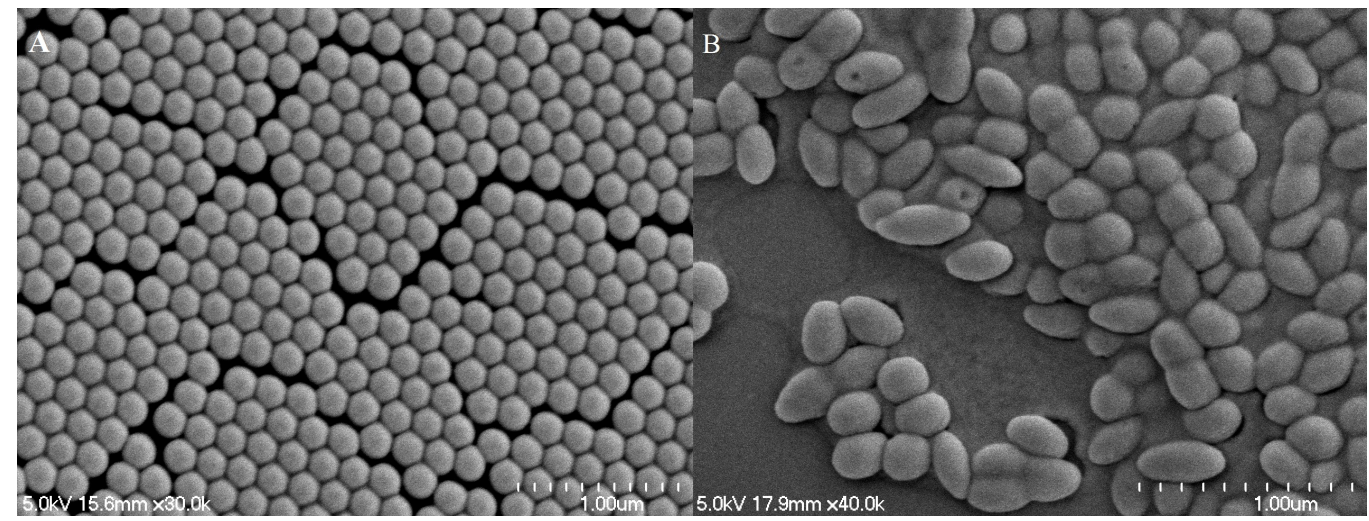

Figure 1: Representative SEM images of spherical (A) and stretched (B) PS particles.

calculation method are provided in Appendix B. After stretching, the major to minor axis ratio was raised from 1:1 to 1.6:1 and the ellipticity was nearly doubled.

The diameter of spherical PS and EPM of spherical and stretched PS were measured as a function of ionic strength and can be seen in Fig. 2. The spherical PS particle diameter was as expected, based on the nominal 200 $\mathrm{nm}$ particle size reported by the manufacturer. The spherical PS diameter remained constant over the ionic strength range investigated, suggesting there was no significant aggregation of the particles. Particle size distributions at each $\mathrm{NaCl}$ concentration investigated can be found in Appendix B.

Both spherical and stretched PS were negatively charged, with the stretched PS carrying a smaller charge (closer to neutral) throughout the entire ionic strength range investigated. Several previous studies have reported a change in EPM between spherical and stretched particles (Seymour et al., 2013; Liu et al., 2010; Salerno et al., 2006; Ho and Ottewill, 1998; Ho et al., 1997). One such study (Seymour et al., 2013), using identical particles to those used here, 


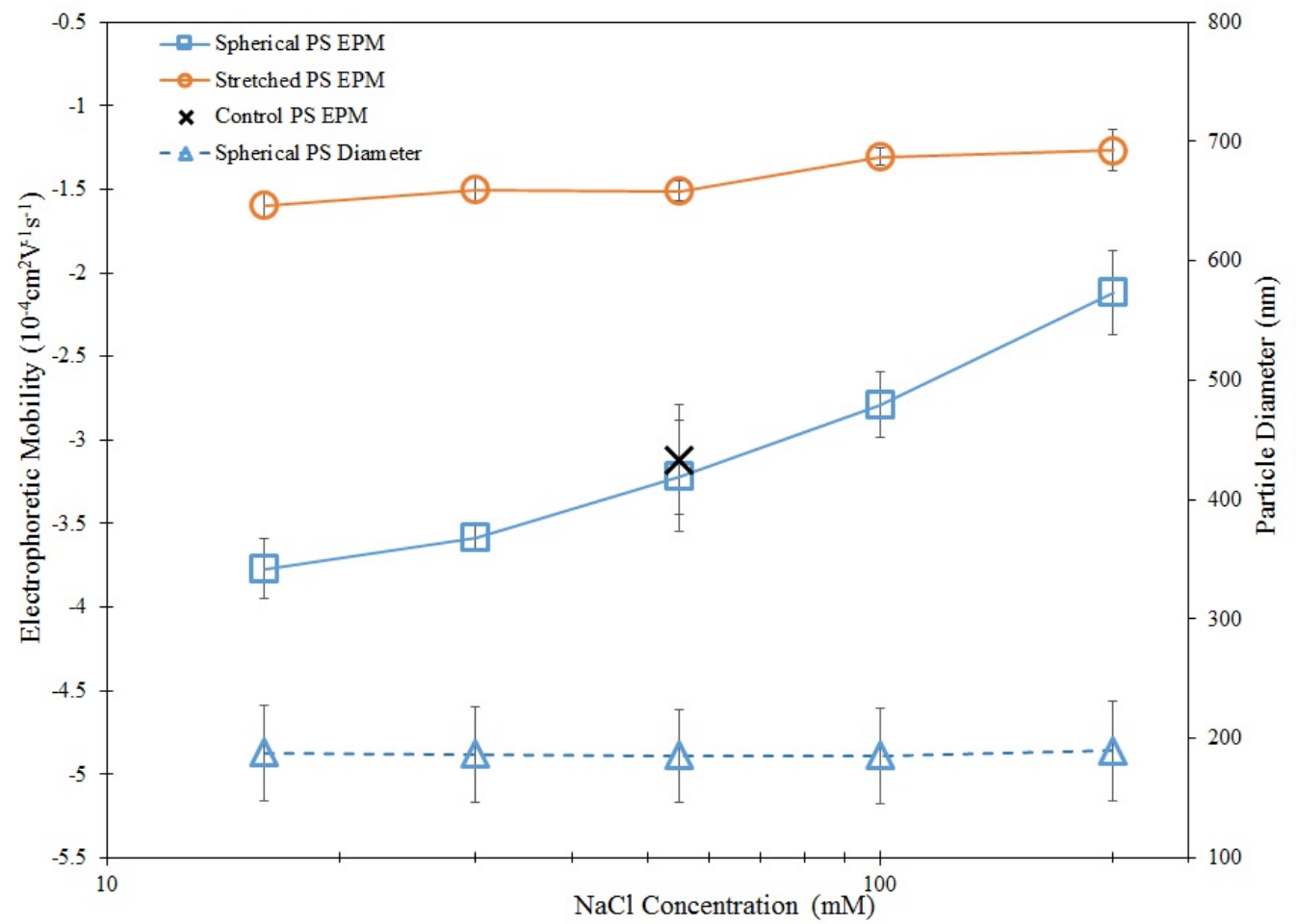

Figure 2: EPM measurements of spherical and stretched PS and particle diameter of spherical PS. The control value refers to spherical PS which were exposed to an identical experimental routine as the stretched PS, without being stretched. Each value was the result of 10 independent measurements and error bars represent standard deviation. 
reported that the EPM of PS particles decreased (closer to neutral) as aspect ratio increased, therefore the sensitivity of EPM to aspect ratio reported here is consistent with previous studies. The sensitivity of EPM to aspect ratio could be a result of altered charge density due to a change in surface area, or altered surface chemistry due to residual chemicals from the particle stretching process (Champion et al., 2007). In order to evaluate which of these possible contributors had a larger effect, a control group of spherical particles was taken through an identical stretching procedure without being stretched, exposing the control group to all the same chemicals. As can be seen in Fig. 2, the EPM of the control particles remained statistically identical to that of the spherical PS particles, similar to the reported control in the aforementioned study (Seymour et al., 2013). This suggests that an altered charge density of the stretched particles is the main contributor to the EPM sensitivity to aspect ratio observed here and elsewhere. The same control group was also used in attachment experiments, to further validate this finding.

The EPM of each depositional surface was also measured and reported in Fig. 3. Each surface was negatively charged throughout the ionic strength range investigated and the EPM of the HHA and alginate surfaces were statistically identical at each ionic strength. These findings are in agreement with previous reports for silica surfaces coated with similar NOM (McNew and LeBoeuf, 2015; Qu et al., 2012). We observed a positive charge for PLLcoated silica, validating it's use to obtain the deposition rate of negatively charged spherical and stretched PS under diffusion-limited conditions (Eq. 2). 


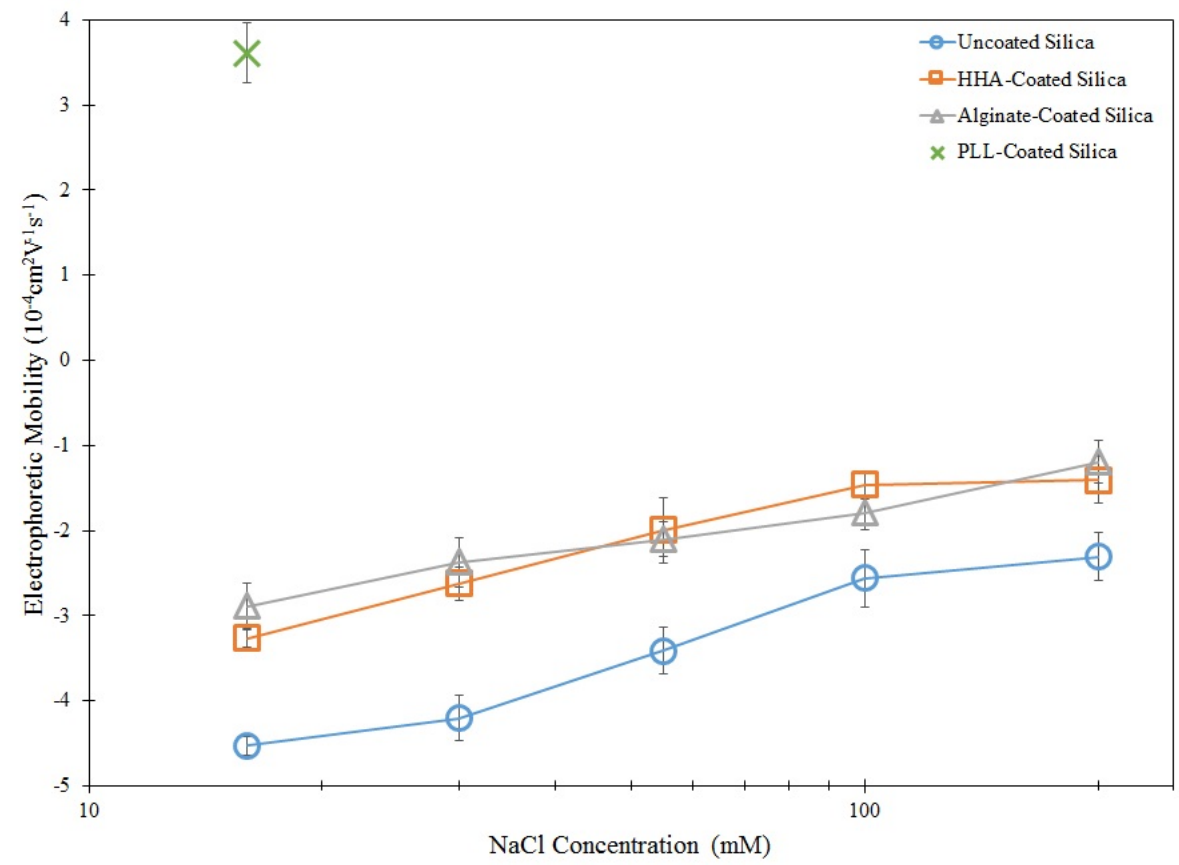

Figure 3: EPM measurements of each depositional surface as a function of ionic strength. Each value was the result of 10 independent measurements and error bars represent standard deviation. 


\subsection{Attachment Results}

For the first time, we report the attachment efficiency of spherical and stretched PS particles onto HHA and alginate coated silica surfaces as a function of ionic strength in Fig. 4. In the presence of the HHA coated silica surface, we observed a higher attachment of spherical than stretched PS at low ionic strengths. At a $\mathrm{NaCl}$ concentration of $100 \mathrm{mM}$, we observed similar attachment values for spherical and stretched PS and above $100 \mathrm{mM} \mathrm{NaCl}$, we observed a higher attachment of stretched than spherical PS. The attachment of the spherical PS was not sensitive to $\mathrm{NaCl}$ concentration, while the attachment of stretched PS showed a steady increase as $\mathrm{NaCl}$ concentration increased. The attachment of the control particles, which were exposed to all the same chemicals as the stretched particles without being stretched, didn't deviate from the attachment of the spherical PS. This confirms that the observed differences in attachment between spherical and stretched particles are due to the differences in particle shape, rather than a surface chemistry alteration as a result of exposure to the chemicals required in the stretching procedure. This control group finding is in agreement with several previous studies (Knappenberger et al., 2015; Aramrak et al., 2013; Salerno et al. 2006), including a study by Seymour et al. (2013) which reported similar deposition rates between spherical PS and control particles, despite observing differences for stretched PS. Attachment of spherical and stretched PS onto the alginate coated silica surface, however, were statistically identical for all ionic strengths investigated, except for a small deviation at $30 \mathrm{mM} \mathrm{NaCl}$.

Total DLVO interaction energy was calculated using the SEI method (Bhattacharjee and Elimelech, 1997) to account for the orientation depen- 

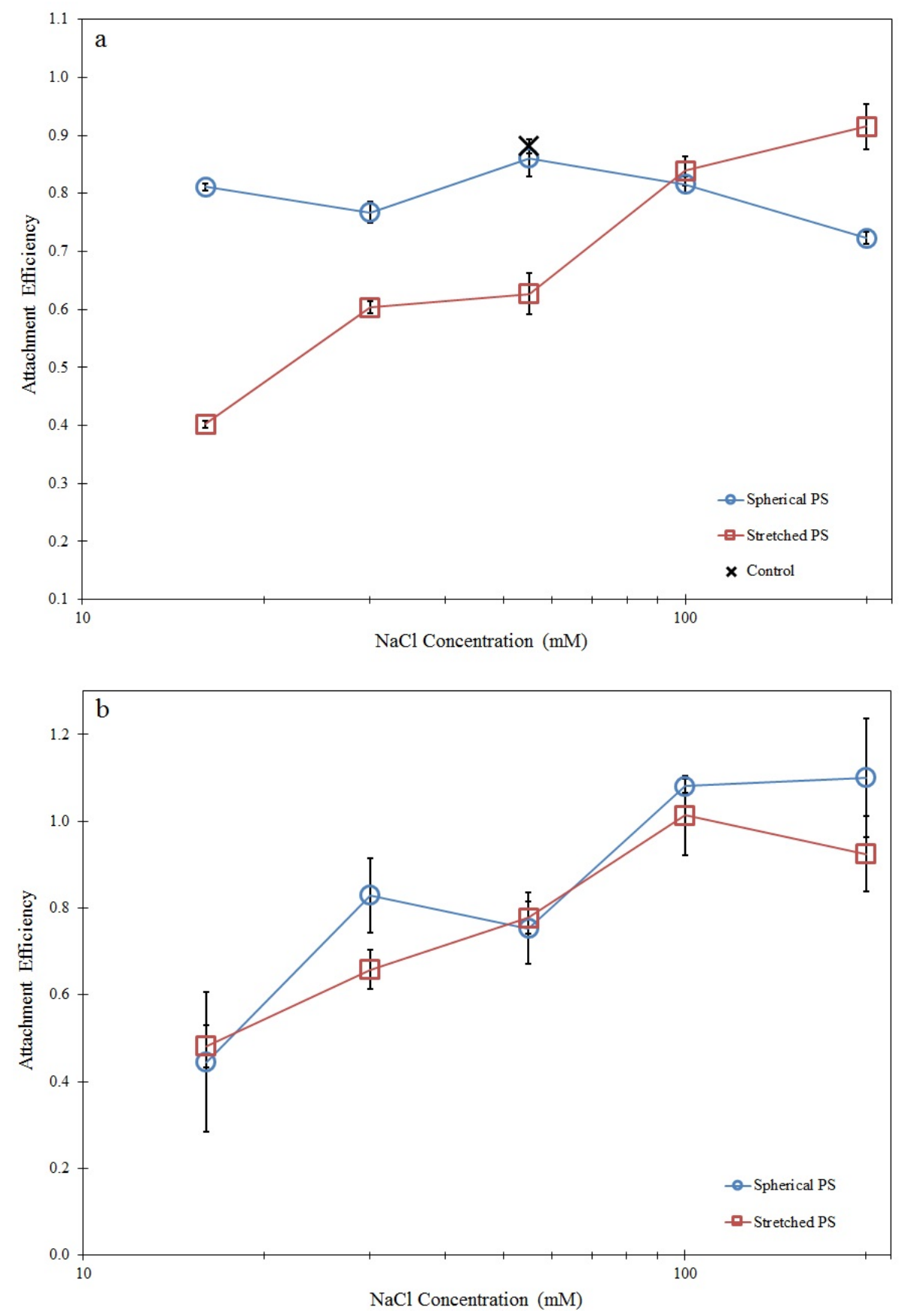

Figure 4: Attachment efficiency of spherical and stretched PS particles onto HHA (a) and alginate (b) coated silica surfaces as a function of ionic strength. Each value was the result of at least 4 independent measurements and error bars represent standard deviation. 

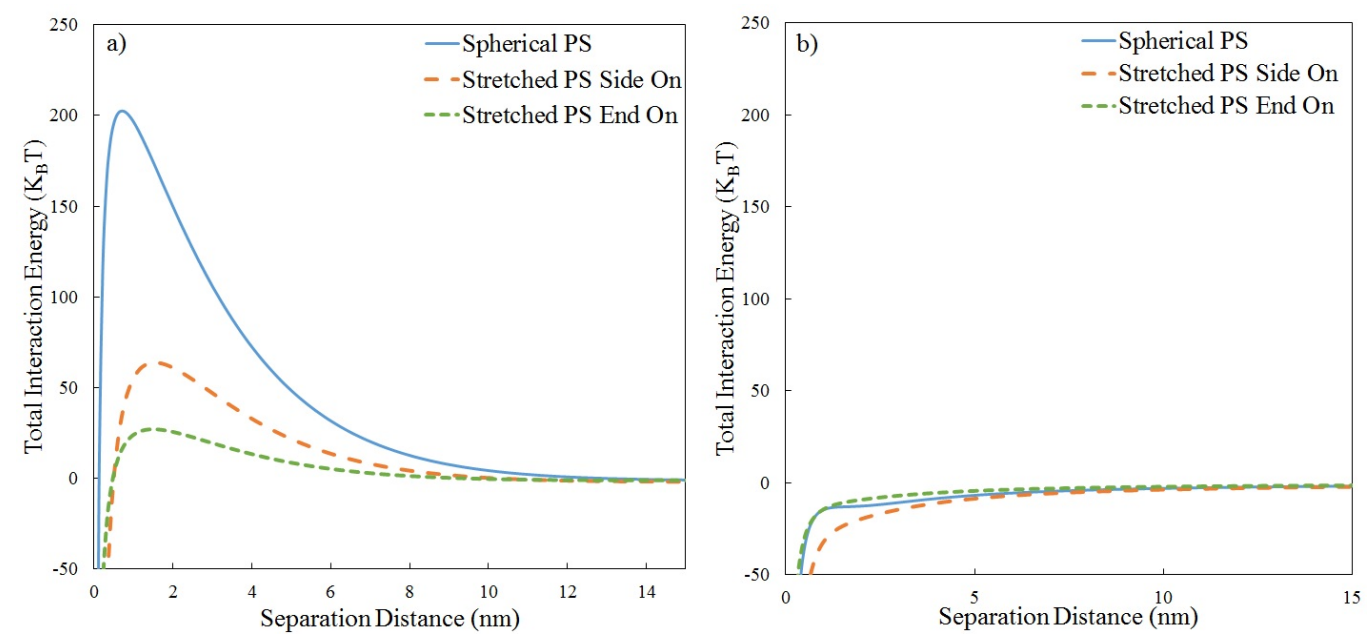

Figure 5: Calculated DLVO interaction energy for the deposition of spherical and stretched PS (side on or end on) onto HHA in the presence of 16 (a) and 200 (b) mM NaCl.

dence of the interaction energy between a flat surface and a spheroidal particle. Details of this calculation method can be found in Appendix D. Total interaction energy for the stretched PS was calculated as the particles approached the surface in two different orientations: major axis parallel to the surface (side on) and major axis perpendicular to the surface (end on). The interaction energy profiles for spherical and stretched PS onto HHA are presented in Fig. 5 in the presence of 16 (a) and 200 (b) $\mathrm{mM} \mathrm{NaCl}$.

As ionic strength increased from $16 \mathrm{mM}$ in Fig. $5 \mathrm{a}$ to $200 \mathrm{mM}$ in $5 \mathrm{~b}$, the electrical double layer repulsion between the negatively charged PS and HHA coated surface is suppressed and the predicted energy barrier to deposition is reduced as a result. The observed attachment trends of stretched PS onto HHA (Fig. 4a) and both spherical and stretched PS onto alginate (Fig. (4b) are in qualitative agreement with this prediction. At $16 \mathrm{mM} \mathrm{NaCl}$, the predicted energy barrier to deposition for spherical PS is much larger than 
that of stretched PS in each particle orientation, due to the less negative (closer to neutral) surface charge of the stretched PS. This is in contradiction to the attachment results presented in Fig. 4a and likely due to surface roughness and steric contributions from the HHA layer, not considered by DLVO theory. Non-DLVO contributions are explored and discussed in detail in the following section.

As illustrated in Fig. 5, the energy barrier to deposition for stretched PS is dependent upon particle orientation. In $16 \mathrm{mM} \mathrm{NaCl}$, the energy barrier to deposition for side on orientation is 2.4 times larger than that of the end on orientation. It has been hypothesized (O’brien and Ward, 1988; Ho and Ottewill, 1998; Loewenberg and Obrien, 1992) that the shape of nonspherical particles will induce a tangential flux of ions near the surface of the particles which, in the case of the rod shaped stretched PS particles, will ultimately result in the accumulation of cations near the ends of the particle. This uneven distribution of surface charge is the cause of the orientation dependent energy barrier observed in Fig. 5. Furthermore, the torques experienced by stretched particles as they turn to energetically favorable positions for attachment can cause a reduction in deposition rates (Seymour et al., 2013). This is discussed in detail in the following section. As the $\mathrm{NaCl}$ concentration is increased to $200 \mathrm{mM} \mathrm{NaCl}$, the energy barrier vanishes due to significant charge screening and the results presented in Fig. 5b suggest that a side on particle orientation allows for a larger attractive energy well than an end on orientation. More detailed DLVO interaction energy information is included in Appendix D. 


\subsection{Surface Topography and Non-DLVO Forces}

The observed attachment trends onto HHA and alginate surfaces are widely different from one another, despite the surfaces possessing statistically identical charges (Fig. 3), therefore the observed differences in attachment behavior are likely due to physical differences of the surfaces. In order to investigate the physical aspect further, both HHA and alginate surfaces were explored using AFM and representative images are reported in Fig. 6 a and b, respectively. The topography of the HHA surface is very heterogeneous, with large peaks extended from the surface, and a root mean squared (RMS) roughness of $2.51 \pm 1.21$. The topography of the alginate surface is smoother, with much fewer peaks extending from the surface relative to HHA, and a RMS roughness of $0.99 \pm 0.26$. The rougher, more extended surface of the HHA layer possesses more voids than the alginate layer, and therefore an increased potential for particle entanglement. The smaller size of the spherical PS particles allow for entanglement in these relatively small voids, while the nearly doubled major axis diameter of the stretched PS hinder particle entanglement within the HHA voids. Furthermore, investigation by the Voigt viscoelastic model (Voinova et al. 1999) estimates that the mass of the HHA layer is more than 5 times larger than the mass of the alginate layer (Fig. B.16). This also suggests that the HHA layer provides a much larger mesh for particle entanglement. These findings are in agreement with two previous studies which reported increased attachment of spherical $\mathrm{nC}_{60}$ onto NOM coated surfaces in the presence of low ionic strength solutions (McNew and LeBoeuf, 2015; Chen and Elimelech, 2008). Particle entanglement, in this manner, is not considered in DLVO theory, and is therefore 


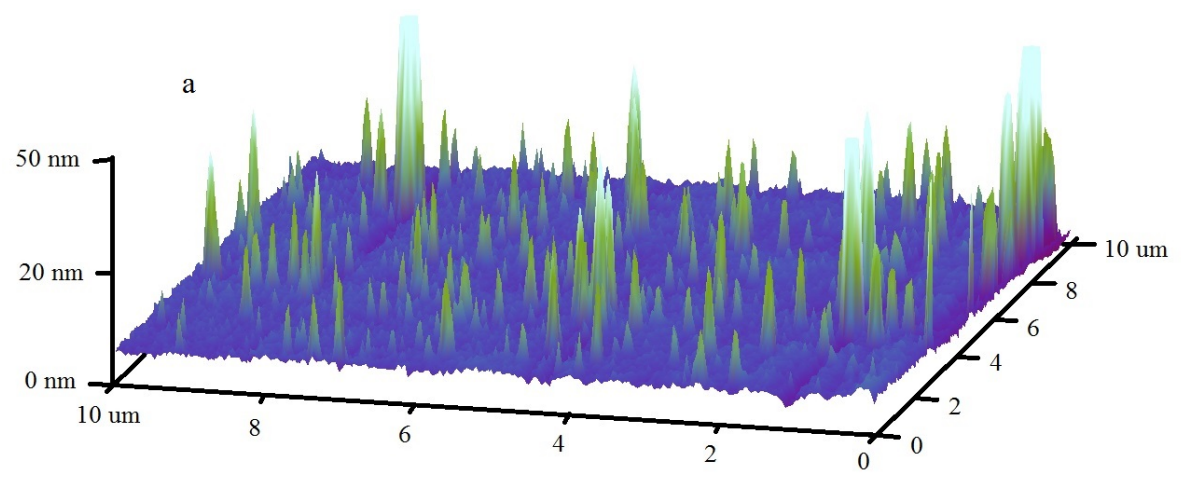

b

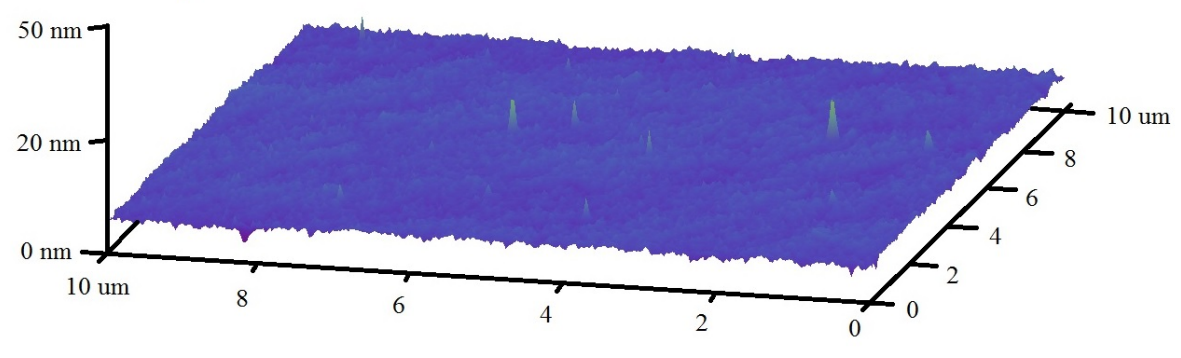

Figure 6: Representative AFM images of HHA (a) and alginate (b) coated silica surfaces.

likely the main contributor to the observed deviation from DLVO theory at low ionic strength. The qualitative agreement to DLVO theory illustrated by the attachment onto alginate, along with the AFM images and measured surface roughness of alginate, however, suggest that the alginate layer did not contribute voids suitable for particle entanglement.

Immobilized macromolecule structure has been reported to depend heavily on the ionic strength of solution (Yuan et al., 2008; Hong and Elimelech, 1997). Furthermore, NOM layers have been reported to condense in the presence of high ionic strength solutions (Chen and Elimelech, 2006, 2008). As ionic strength increases, the HHA layer condenses, induced by increased inter- and intra-molecular crosslinks, forming a smoother, less extended sur- 
face with fewer voids for particle entanglement. Since the condensed surface has a lower potential for particle entanglement, the particle entanglement effect seen at low ionic strength diminishes as ionic strength increases. Though spherical PS particles experience a reduced energy barrier to deposition as ionic strength increases (Fig. 5), this effect is offset by reduced particle entanglement, induced by HHA layer compaction, leading to no significant net change in attachment and a spherical PS attachment trend which is relatively insensitive to ionic strength. Since stretched PS particles experience less particle entanglement due to their enlarged major axis, they are free to experience the reduced energy barrier and the stretched PS attachment trend increases with ionic strength, as predicted by DLVO theory. Two previous studies have reported reduced attachment of spherical particles onto NOM layers as ionic strength increased (McNew and LeBoeuf, 2015; Chen and Elimelech, 2008), however none have yet illustrated the dependency on aspect ratio reported in this study. Though all experiments in this study were performed in the presence of monovalent salt solutions, in our previous study (McNew and LeBoeuf, 2015) we reported a similar HHA layer compaction in the presence of both mono and divalent cations $\left(\mathrm{Na}^{+}\right.$and $\left.\mathrm{Ca}^{2+}\right)$. Whether or not this finding extends to variable particle shape, however, is not yet known.

Though stretched PS particles exhibit a lower (closer to neutral) surface charge (Fig. 2), we observe statistically similar attachment between stretched and spherical PS onto the smooth alginate layer throughout the ionic strength range investigated (Fig. 4b). The reason for this deviation from DLVO lies within the orientation dependent interaction energy of the stretched PS. The 
stretched PS experience torques to align the particles into an energetically favorable orientation for deposition (O'brien and Ward, 1988; Ho and Ottewill, 1998; Loewenberg and Obrien, 1992). These rotational forces and induced direction changes cause the stretched PS particles to follow an oscillatory flow path with respect to the depositional surface, as observed in several studies on the flow trajectory of submicrometer, non-spherical particles (Gavze and Shapiro, 1995; Blaser, 2002; Decuzzi and Ferrari, 2006). The flow path of the spherical particles within these studies, however, was observed to be parallel to the surface. In this manner, the larger hydrodynamic forces experienced by the stretched PS led to a reduction in deposition rate, which resulted in attachment values comparable to that of the more electrostatically repulsive spherical PS.

\subsection{Interpretation of Energy Dissipation}

The dissipation parameter is a dimensionless quantity which can be used to infer about the viscoelastic properties of the mass coupled to the sensor Kananizadeh et al. 2016; Dixon, 2008). Rigid films result in a longer decay in the oscillatory energy and therefore lower values of the dissipation parameter while soft or viscoelastic films result in much faster oscillatory energy dissipation, due to the poor coupling of the soft material to the stiff sensor, and therefore higher values of the dissipation parameter. Representative QCM-D deposition data can be seen in Fig. 7 for four different cases: (a) spherical PS onto PLL, (b) spherical PS onto HHA, (c) stretched PS onto PLL, and (d) stretched PS onto HHA. All experiments took place in $30 \mathrm{mM}$ $\mathrm{NaCl}$ and overtones $3,5,7,9,11$, and 13 are displayed for both frequency and dissipation. 

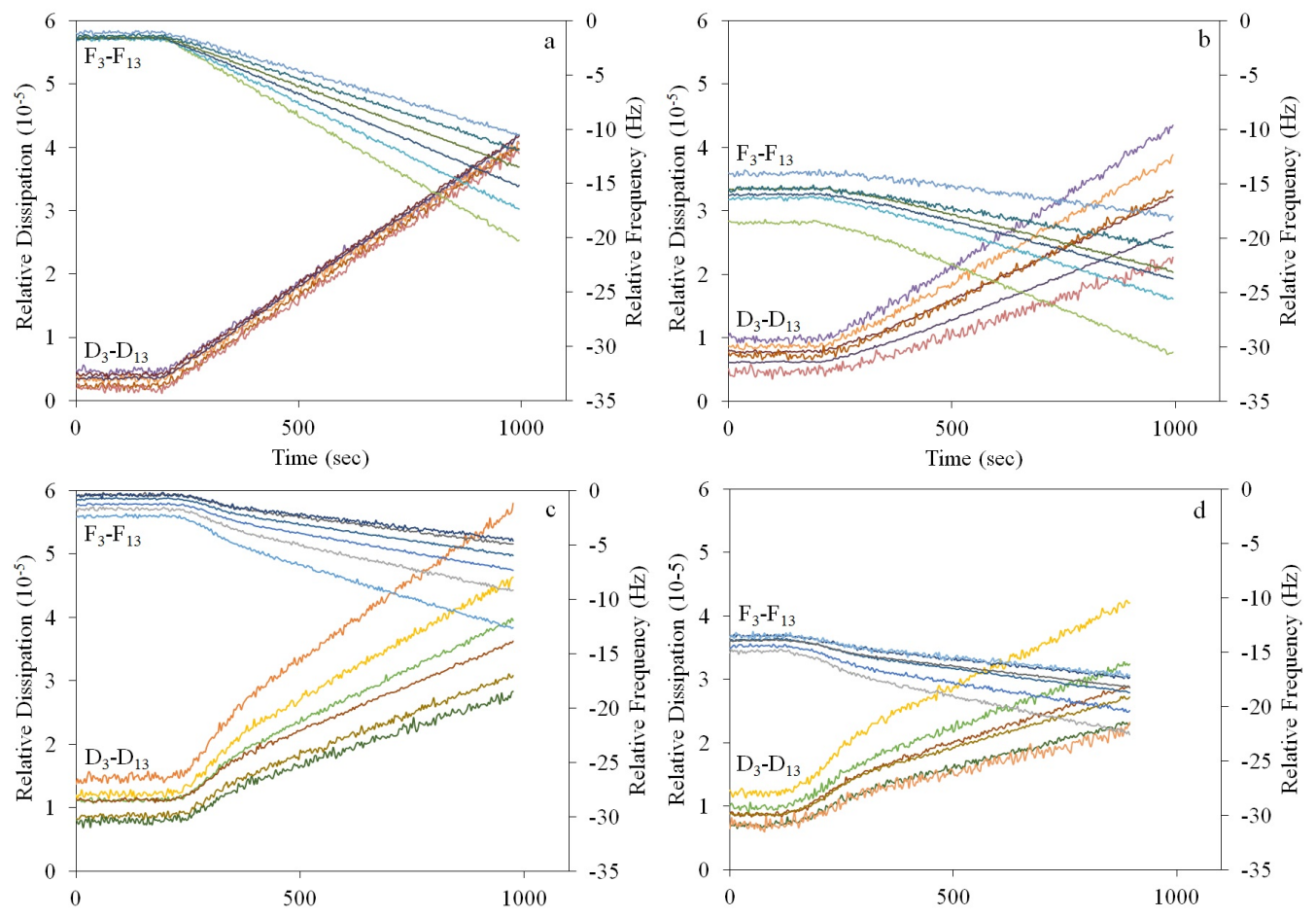

Figure 7: Representative QCM-D deposition data for spherical PS onto PLL (a), spherical PS onto HHA (b), stretched PS onto PLL (c), and stretched PS onto HHA (d). All experiments took place in $30 \mathrm{mM} \mathrm{NaCl}$. 
A significant increase in dissipation was observed $(>5 \% \Delta \mathrm{D})$ for each case presented in Fig. 7, suggesting that both spherical and stretched PS particles attached to each surface in a soft, viscoelastic layer (Kananizadeh et al., 2016, Reviakine et al., 2011; Dixon, 2008). As the spherical PS attached to the PLL surface (Fig. 7a), no additional spread in the overtones of dissipation was observed, suggesting that the particles formed a homogenous, soft layer (Reviakine et al., 2011; Dixon, 2008). A homogenous layer is expected in this case, due to the spherical shape of the particles and their resulting independence upon particle orientation. As the stretched PS attached to the PLL surface (Fig. 7c) however, a very pronounced spread of the overtones of dissipation is observed, suggesting the formation of a heterogenous, soft film. Due to their altered aspect ratio, the stretched PS particles attach to the surface in various configurations, leading to an increase in surface heterogeneity and roughness, resulting in a larger dissipation increase and much larger spread of overtones. As expected for the rougher and more extended HHA layer, attachment of both spherical (Fig. 7b) and stretched PS (Fig. 7 $\mathrm{d}$ ) resulted in a heterogeneous, soft layer. Despite attaching at a lower rate (Fig. 4), the stretched PS resulted in a similar increase and spread of dissipation overtones. This can again be attributed to the altered aspect ratio of the stretched particles, resulting in various attachment orientations.

\section{Conclusions}

In this study, we showed that the attachment of colloidal particles onto NOM-coated surfaces can be greatly affected by the shape of the particles, depending on ionic strength and the type of NOM coating. The altered charge 
distribution of the stretched PS induced an orientation dependent interaction energy between the particles and the surface, which led to stronger hydrodynamic forces and an oscillatory flow path near the surface, resulting in a reduction in the observed attachment. In the presence of the alginate surface, the attachment of both spherical and stretched PS qualitatively agreed with DLVO theory. In the presence of the HHA surface, however, deviations from DLVO theory were observed. At low ionic strengths, spherical PS attached to HHA at a much higher rate than stretched PS. This deviation from DLVO theory can be explained by the increased surface heterogeneity and roughness observed for the HHA surface. More voids present in the HHA layer allowed for a higher potential of spherical PS particle entanglement, while the enlarged major axis of the stretched particles hindered their ability to become entangled in the extended HHA surface. As ionic strength increased, the HHA layer condensed and the potential for spherical particle entanglement is reduced.

Complex interactions exist between colloidal particles and environmentally relevant surfaces and, based on the findings presented in this study, it is clear that the shape of colloidal particles can play a major role in such interactions. If similarly complex interactions exist for other types of environmentally relevant surfaces, particle shape may greatly affect the environmental fate and transport of colloidal particles. A complete understanding of the role of particle shape will improve the accuracy of colloidal particle transport predictions, aiding in the assessment of risk and potential exposure pathways. 


\section{Acknowledgements}

This study was supported by the National Science Foundation Award Nos. 1133528 and 1133280, the Vanderbilt University Discovery Grant Program, and the Vanderbilt University Department of Civil and Environmental Engineering.

\section{References}

Nelson Akaighe, Sean W. Depner, Sarbajit Banerjee, and Mary Sohn. Transport and deposition of suwannee river humic acid/natural organic matter formed silver nanoparticles on silica matrices: The influence of solution ph and ionic strength. Chemosphere, 92(4):406-412, 2013.

Surachet Aramrak, Markus Flury, James B Harsh, Richard L Zollars, and Howard P Davis. Does colloid shape affect detachment of colloids by a moving air-water interface? Langmuir, 29(19):5770-5780, 2013.

D.L. Balkwill, J.K. Fredrickson, and J.M. Thomas. Vertical and horizontal variations in the physiological diversity of the aerobic chemoheterotrophic bacterial microflora in deep southeast coastal plain subsurface sediments. Applied and Environmental Microbiology, 55(5):1058-1065, 1989.

Ruth E. Baltus, Appala Raju Badireddy, Wendong Xu, and Shankararaman Chellam. Analysis of configurational effects on hindered convection of nonspherical bacteria and viruses across microfiltration membranes. Industrial Ef Engineering Chemistry Research, 48(5):2404-2413, 2008. 
Subir Bhattacharjee and Menachem Elimelech. Surface element integration: a novel technique for evaluation of dlvo interaction between a particle and a flat plate. Journal of Colloid and Interface Science, 193(2):273-285, 1997.

Stefan Blaser. Forces on the surface of small ellipsoidal particles immersed in a linear flow field. Chemical Engineering Science, 57(3):515-526, 2002.

Julie A. Champion, Yogesh K. Katare, and Samir Mitragotri. Making polymeric micro- and nanoparticles of complex shapes. Proceedings of the $\mathrm{Na}$ tional Academy of Sciences, 104(29):11901-11904, 2007.

Xiaojun Chang and Dermont C. Bouchard. Multiwalled carbon nanotube deposition on model environmental surfaces. Environmental Science \& Technology, 47(18):10372-10380, 2013.

Kai Loon Chen and Menachem Elimelech. Aggregation and deposition kinetics of fullerene (c-60) nanoparticles. Langmuir, 22(26):10994-11001, 2006.

Kai Loon Chen and Menachem Elimelech. Interaction of fullerene (c-60) nanoparticles with humic acid and alginate coated silica surfaces: Measurements, mechanisms, and environmental implications. Environmental Science \&6 Technology, 42(20):7607-7614, 2008.

Indranil Chowdhury, Matthew C. Duch, Nikhita D. Mansukhani, Mark C. Hersam, and Dermont Bouchard. Deposition and release of graphene oxide nanomaterials using a quartz crystal microbalance. Environmental Science E Technology, 48(2):961-969, 2014a. 
Indranil Chowdhury, Matthew C. Duch, Nikhita D. Mansukhani, Mark C. Hersam, and Dermont Bouchard. Interactions of graphene oxide nanomaterials with natural organic matter and metal oxide surfaces. Environmental Science \& Technology, 48(16):9382-9390, 2014b.

P Decuzzi and Mauro Ferrari. The adhesive strength of non-spherical particles mediated by specific interactions. Biomaterials, 27(30):5307-5314, 2006.

C. Degueldre, H.R. Pfeiffer, W. Alexander, B. Wernli, and R. Bruetsch. Colloid properties in granitic groundwater systems. i: Sampling and characterisation. Applied Geochemistry, 11(5):677-695, 1996.

Rossane C. DeLapp, Eugene J. LeBoeuf, Jie Chen, and Baohua Gu. Advanced thermal characterization of fractionated natural organic matter. Journal of Environmental Quality, 34(3):842-853, 2005.

B.V. Derjaguin. Theory of the stability of strongly charged lyophobic sols and the adhesion of strongly charged particles in solutions of electrolytes. Acta Physicochim. USSR, 14:633-662, 1941.

Matthew C Dixon. Quartz crystal microbalance with dissipation monitoring: enabling real-time characterization of biological materials and their interactions. Journal of Biomolecular Techniques, 19(3):151-158, 2008.

Menachem Elimelech, Xiadong Jia, John Gregory, and Richard Williams. Particle deposition $\&$ aggregation: measurement, modelling and simulation. Butterworth-Heinemann, 1998. 
Menachem Elimelech, John Gregory, and Xiadong Jia. Particle deposition and aggregation: measurement, modelling and simulation. ButterworthHeinemann, 2013.

E Gavze and M Shapiro. Nonspherical particles in a shear flow near a solid wall. Journal of Aerosol Science, 26(6):1021, 1995.

Peter Hirsch and Ellen Rades-Rohkohl. Microbial colonization of aquifer sediment exposed in a groundwater well in northern germany. Applied and Environmental Microbiology, 56(10):2963-2966, 1990.

C.C. Ho and R.H. Ottewill. Investigation of the charge distribution of ellipsoidal particles. Colloids and Surfaces A: Physicochemical and Engineering Aspects, 141(1):29-35, 1998.

C.C. Ho, R.H. Ottewill, and L. Yu. Examination of ellipsoidal polystyrene particles by electrophoresis. Langmuir, 13(7):1925-1930, 1997.

R. Hogg, T.W. Healy, and D.W. Fuerstenau. Mutual coagulation of colloidal dispersions. Transactions of the Faraday Society, 62:1638-1651, 1966.

Seungkwan Hong and Menachem Elimelech. Chemical and physical aspects of natural organic matter (nom) fouling of nanofiltration membranes. Journal of Membrane Science, 132(2):159-181, 1997.

Robert J. Hunter. Zeta potential in colloid science: principles and applications. Academic press, 2013.

X. Jiang, M. Tong, H. Li, and K. Yang. Deposition kinetics of zinc oxide 
nanoparticles on natural organic matter coated silica surfaces. Journal of Colloid and Interface Science, 350(2):427-434, 2010.

Negin Kananizadeh, Charles Rice, Jaewoong Lee, Keith B Rodenhausen, Derek Sekora, Mathias Schubert, Eva Schubert, Shannon Bartelt-Hunt, and Yusong Li. Combined quartz crystal microbalance with dissipation (qcm-d) and generalized ellipsometry (ge) to characterize the deposition of titanium dioxide nanoparticles on model rough surfaces. Journal of Hazardous Materials, 2016.

Thorsten Knappenberger, Surachet Aramrak, and Markus Flury. Transport of barrel and spherical shaped colloids in unsaturated porous media. Journal of Contaminant Hydrology, 180:69-79, 2015.

Qiang Liu, Volha Lazouskaya, Qingxiang He, and Yan Jin. Effect of particle shape on colloid retention and release in saturated porous media. Journal of Environmental Quality, 39(2):500-508, 2010.

M Loewenberg and RW Obrien. The dynamic mobility of nonspherical particles. Journal of Colloid and Interface Science, 150(1):158-168, 1992.

Coy P. McNew and Eugene J. LeBoeuf. The role of attached phase soil and sediment organic matter physicochemical properties on fullerene ( $\left.\mathrm{nc}_{60}\right)$ attachment. Chemosphere, 139:609-616, 2015.

Andre Nel, Tian Xia, Lutz Mädler, and Ning Li. Toxic potential of materials at the nanolevel. Science, 311(5761):622-627, 2006.

RW O'brien and Derek Noel Ward. The electrophoresis of a spheroid with 
a thin double layer. Journal of Colloid and Interface Science, 121(2):402413, 1988.

Xiaolei Qu, Pedro JJ Alvarez, and Qilin Li. Impact of sunlight and humic acid on the deposition kinetics of aqueous fullerene nanoparticles (nc60). Environmental Science \&5 Technology, 46(24):13455-13462, 2012.

Ilya Reviakine, Diethelm Johannsmann, and Ralf P Richter. Hearing what you cannot see and visualizing what you hear: interpreting quartz crystal microbalance data from solvated interfaces. Analytical Chemistry, 83(23): 8838-8848, 2011.

Michael B. Salerno, Matt Flamm, Bruce E. Logan, and Darrell Velegol. Transport of rodlike colloids through packed beds. Environmental Science \& Technology, 40(20):6336-6340, 2006.

Günter Sauerbrey. Verwendung von schwingquarzen zur wagung dunner schichten und zur mikrowagung. Z. Phys, 155:206-222, 1959.

Megan B. Seymour, Gexin Chen, Chunming Su, and Yusong Li. Transport and retention of colloids in porous media: does shape really matter? Environmental Science $\&$ Technology, 47(15):8391-8398, 2013.

Yun Shen, Hyunjung Kim, Meiping Tong, and Qingyun Li. Influence of solution chemistry on the deposition and detachment kinetics of rna on silica surfaces. Colloids and Surfaces B: Biointerfaces, 82(2):443-449, 2011.

Beng Joo Reginald Thio, Dongxu Zhou, and Arturo A. Keller. Influence of natural organic matter on the aggregation and deposition of titanium 
dioxide nanoparticles. Journal of Hazardous Materials, 189(1):556-563, 2011.

Meiping Tong, Jiali Ding, Yun Shen, and Pingting Zhu. Influence of biofilm on the transport of fullerene (c 60) nanoparticles in porous media. Water Research, 44(4):1094-1103, 2010.

E.J.W. Verwey. Theory of the stability of lyophobic colloids. The Journal of Physical Chemistry, 51(3):631-636, 1947.

Marina V. Voinova, M. Rodahl, M. Jonson, and B. Kasemo. Viscoelastic acoustic response of layered polymer films at fluid-solid interfaces: continuum mechanics approach. Physica Scripta, 59(5):391, 1999.

Yingying Wang, Frederik Hammes, Marcel Düggelin, and Thomas Egli. Influence of size, shape, and flexibility on bacterial passage through micropore membrane filters. Environmental Science \& Technology, 42(17):6749-6754, 2008.

C.H. Ward, W. Giger, and P.L. McCarty. Groundwater Quality (Environmental Science and Technology Series). John Wiley \& Sons Inc, 1985. ISBN 0471815977.

Thomas H. Weiss, Aaron L. Mills, George M. Hornberger, and Janet S. Herman. Effect of bacterial cell shape on transport of bacteria in porous media. Environmental Science \& Technology, 29(7):1737-1740, 1995.

Lei Wu, Bin Gao, Yuan Tian, Rafael Mun oz Carpena, and Kirk J Zigler. Dlvo interactions of carbon nanotubes with isotropic planar surfaces. Langmuir, 29(12):3976-3988, 2013. 
Peng Yi and Kai Loon Chen. Influence of surface oxidation on the aggregation and deposition kinetics of multiwalled carbon nanotubes in monovalent and divalent electrolytes. Langmuir, 27(7):3588-3599, 2011.

Baoling Yuan, Mai Pham, and Thanh H. Nguyen. Deposition kinetics of bacteriophage ms2 on a silica surface coated with natural organic matter in a radial stagnation point flow cell. Environmental Science \& Technology, 42(20):7628-7633, 2008.

\section{Appendix A. Detailed Experimental Information}

\section{Appendix A.1. Particle Stretching Details}

The method described here is based on a procedure previously published by Champion et al. (2007). First, 10\% (wt/vol) polyvinyl alcohol (PVA, Sigma Aldrich) solution was introduced into $80{ }^{\circ} \mathrm{C}$ Milli-Q water, and stirred for 30 minutes to achieve complete dissolution of PVA. $100 \mathrm{mg}$ glycerol (Sigma Aldrich) and $2.5 \mathrm{~mL}$ of $1 \%$ solid content $(0.0625 \%$ (wt/vol) particle/water) PS spheres were added to the PVA solution. The solution mixture was then poured onto a $12 \times 12 \mathrm{~cm}$ clean film plate and was set to dry for 24 hours to form a thin film. The film was then cut into four pieces and subsequently placed in a stretching apparatus (Fig. A.8). The stretching apparatus was submerged in a $125^{\circ} \mathrm{C}$ mineral oil (Fisher Scientific) bath for 5 minutes and stretched to a 2:1 ratio. This temperature is close to the glass transition temperature of polystyrene particles, which allows the particles to stretch to the desired ratio. The stretched film was then cooled to room temperature and cut from the apparatus. After being cleaned by $100 \%$ isopropyl 
alcohol (IPA, Fisher Scientific) for at least two hours, the film was dissolved ${ }_{624}$ in $70{ }^{\circ} \mathrm{C}$ water for several hours. Finally, the stretched PS particles were ${ }_{626}$ Centrifuge 5840R).

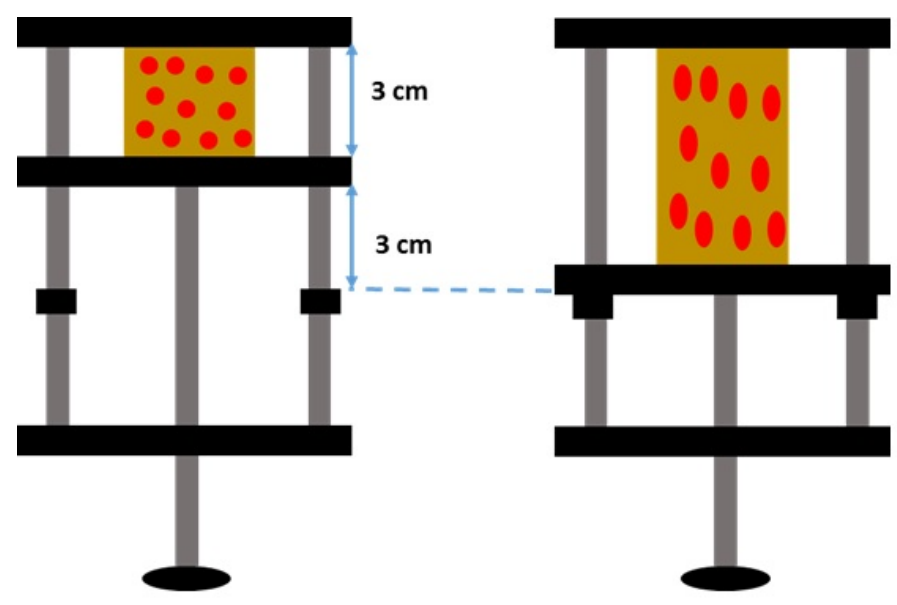

Figure A.8: Diagram of the particle stretching apparatus before (a) and after (b) stretching. 


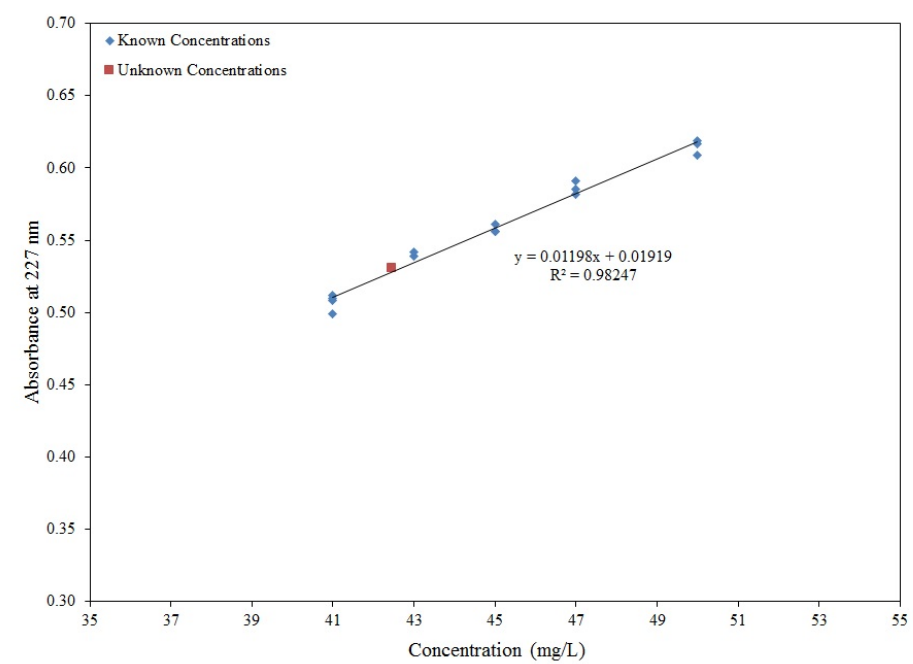

Figure A.9: Calibration curve used in the determination of particle concentration following the stretching procedure.

\section{Appendix A.2. QCM-D Experimental Protocol}

A representative QCM-D experiment is presented in Fig. A.10. A baseline was first collected in a HEPES solution (10 mM HEPES, $100 \mathrm{mM} \mathrm{NaCL}$ ). The signal was considered stable when the third overtone of frequency $\left(f_{3}\right)$ failed to drift more than $0.3 \mathrm{~Hz}$ over the course of 10 minutes. Then, a layer of positively charged PLL was constructed on the silica surface by flowing a solution of $0.1 \mathrm{~g} \mathrm{~L}^{-1}$ PLL, $10 \mathrm{mM}$ HEPES, and $100 \mathrm{mM} \mathrm{NaCl}$ until the signal stabilized. The PLL layer was then washed in the HEPES solution to remove any unbound PLL from the surface. A $1 \mathrm{mM} \mathrm{NaCl}$ solution was then introduced until a stable signal was reached, followed by a $0.1 \mathrm{~g} \mathrm{~L}^{-1}$ HHA or alginate solution containing $1 \mathrm{mM} \mathrm{NaCl}$. The negatively charged HHA or alginate readily adsorbed to the oppositely charged PLL surface, forming a second layer. Once the signal stabilized, the HHA or alginate 
640 layer was washed in $1 \mathrm{mM} \mathrm{NaCl}$ to remove any unbound particles and then ${ }_{641}$ a solution of the ionic strength of interest was introduced. Once the signal 642 stabilized, the stock suspension of spherical or stretched PS was diluted to 6 ${ }_{643} \mathrm{mg} \mathrm{L}^{-1}$ with a measured amount of concentrated $\mathrm{NaCl}$ solution to form the 644 same ionic strength and then immediately introduced into the measurement 645 chamber. As the PS suspension flowed across the sensor surface, the first 120 646 seconds of the drop in the third overtone of frequency were used to calculate 647 the initial deposition rates for each set of experimental conditions. 


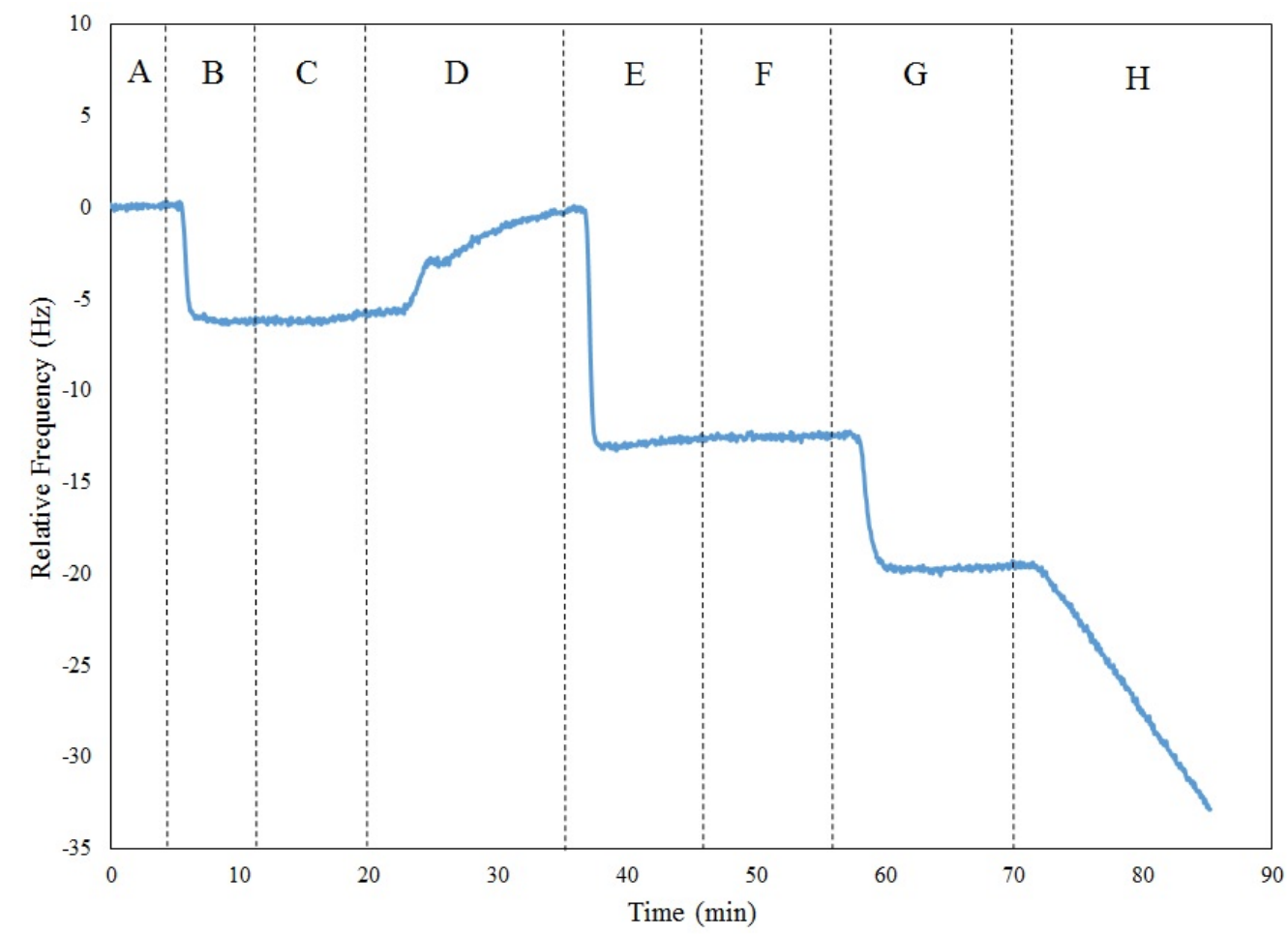

Figure A.10: Representative attachment experiment. A baseline is collected in HEPES (A) before PLL is adsorbed to the surface (B), followed by a rinse in HEPES (C). $1 \mathrm{mM}$ $\mathrm{NaCl}$ is introduced (D) before HHA or alginate is adsorbed to the surface (E) and rinsed with $1 \mathrm{mM} \mathrm{NaCl}(\mathrm{F})$. Finally, the ionic strength of interest is introduced $(\mathrm{G})$ and once a baseline is reached, stretched or spherical PS particles are introduced in a solution of the same ionic strength $(\mathrm{H})$. The third overtone of frequency is displayed. 

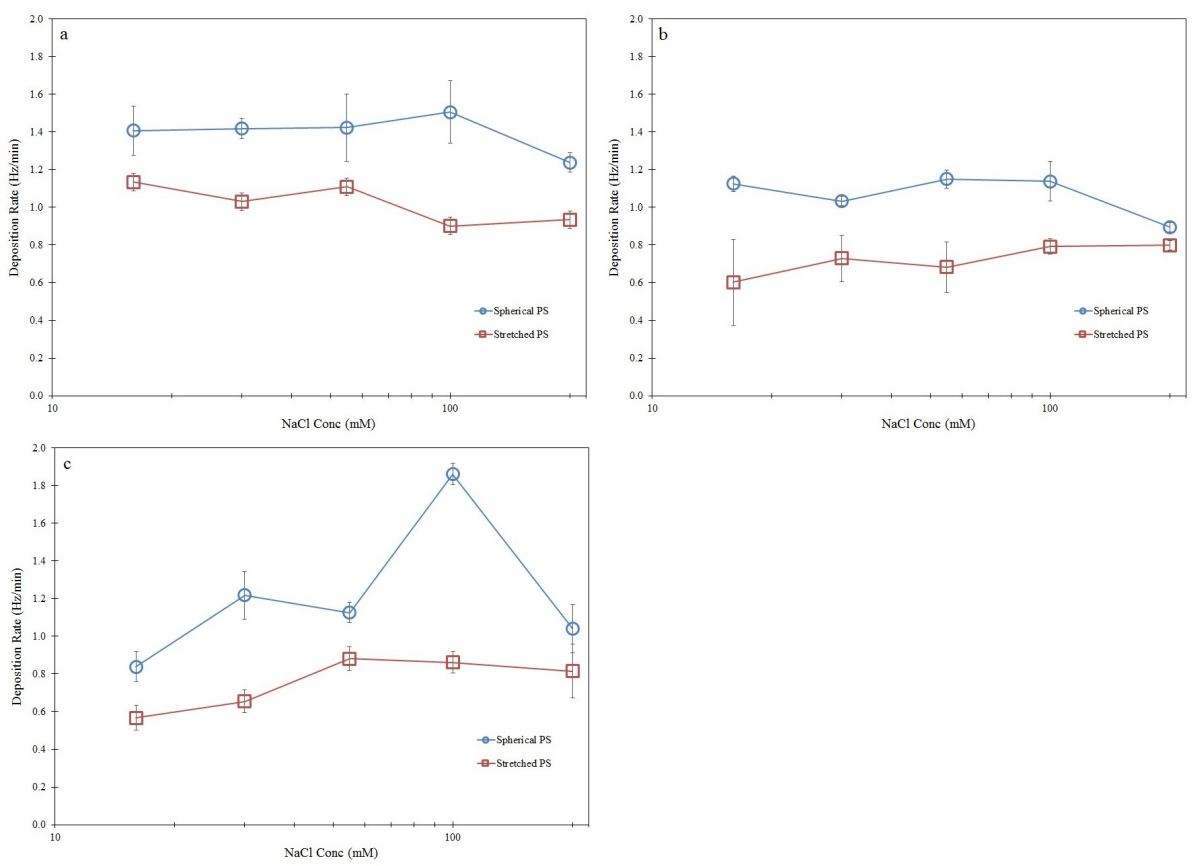

Figure A.11: The deposition rates of spherical and stretched PS onto bare (a), HHA-coated (b), and alginate-coated (c) silica at each $\mathrm{NaCl}$ concentration investigated. 
649 Appendix B. Detailed Characterization Information

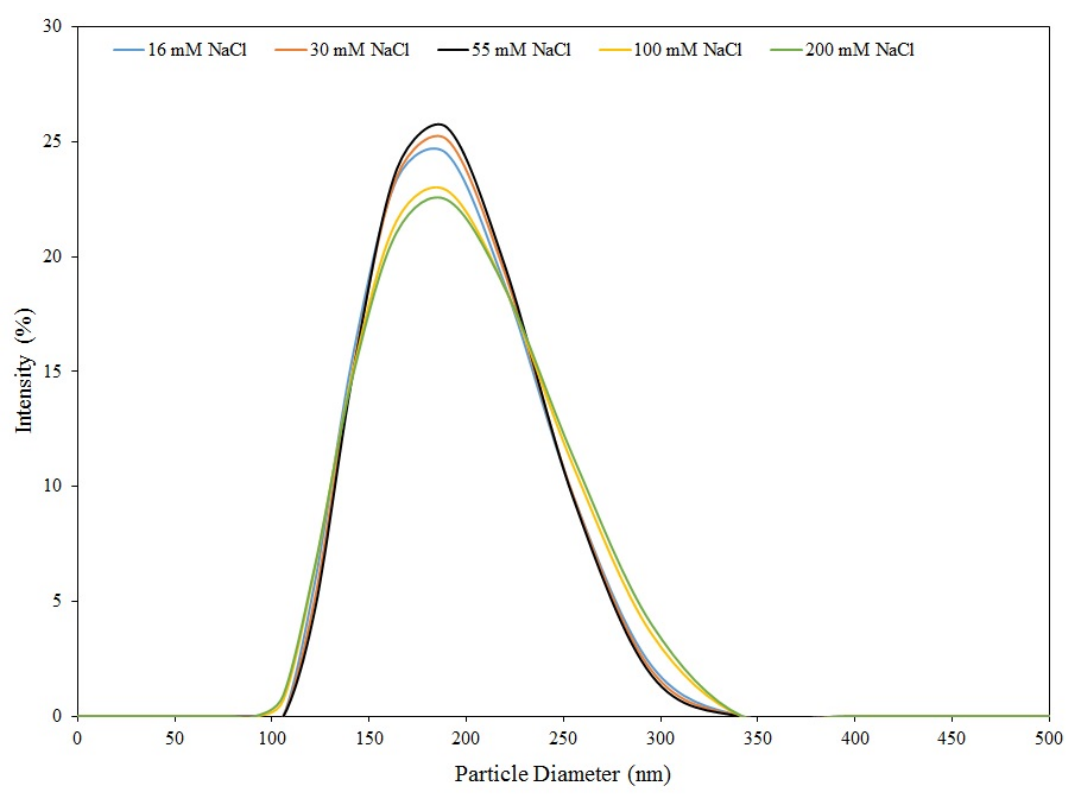

Figure B.12: Particle size distributions of spherical PS at each $\mathrm{NaCl}$ concentration investigated, determined by DLS. 


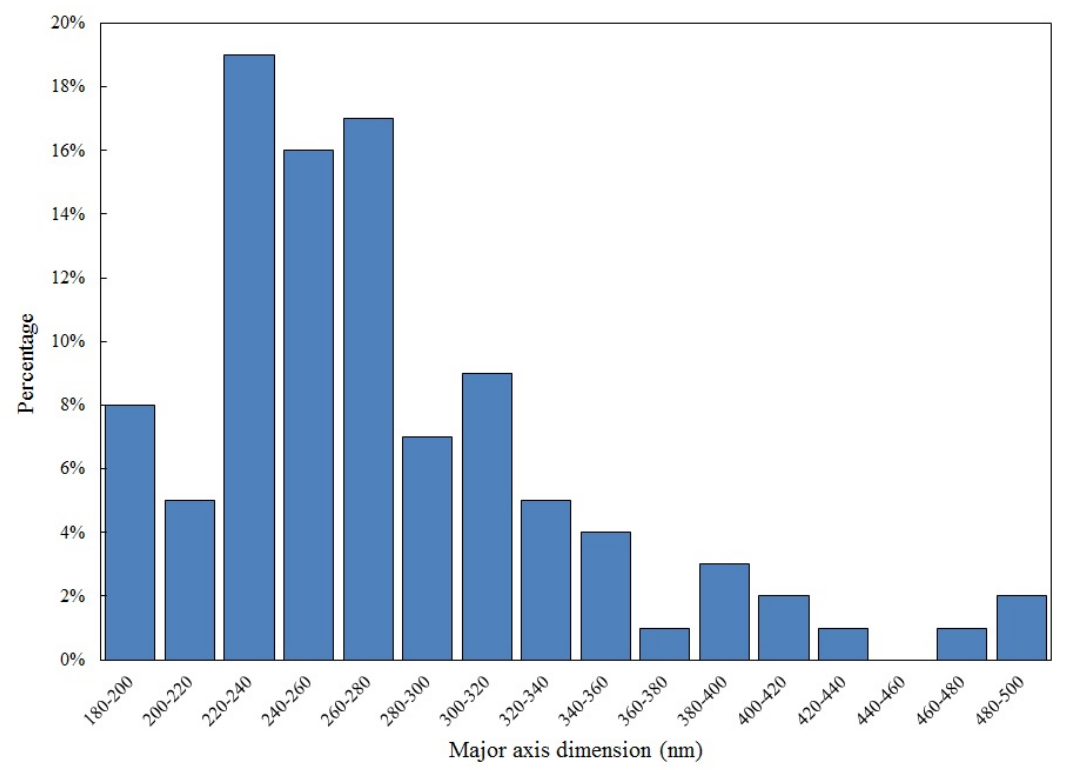

Figure B.13: Summary of the major axis dimensions of stretched PS.

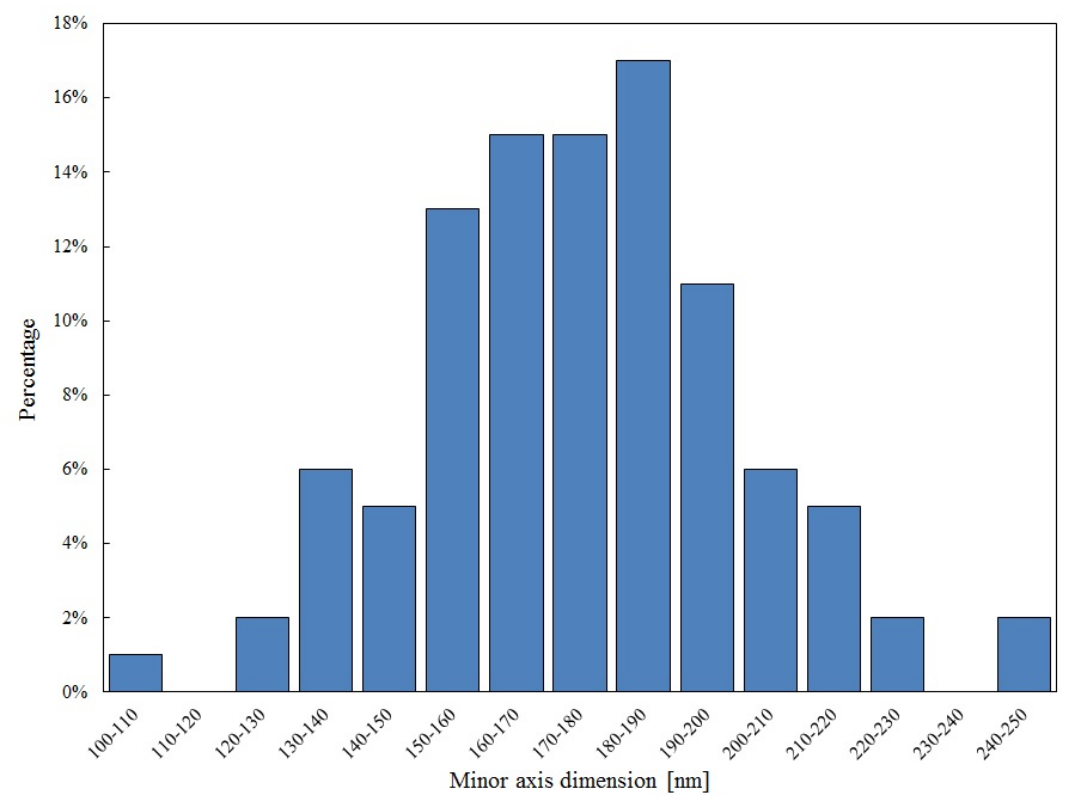

Figure B.14: Summary of the minor axis dimensions of stretched PS. 


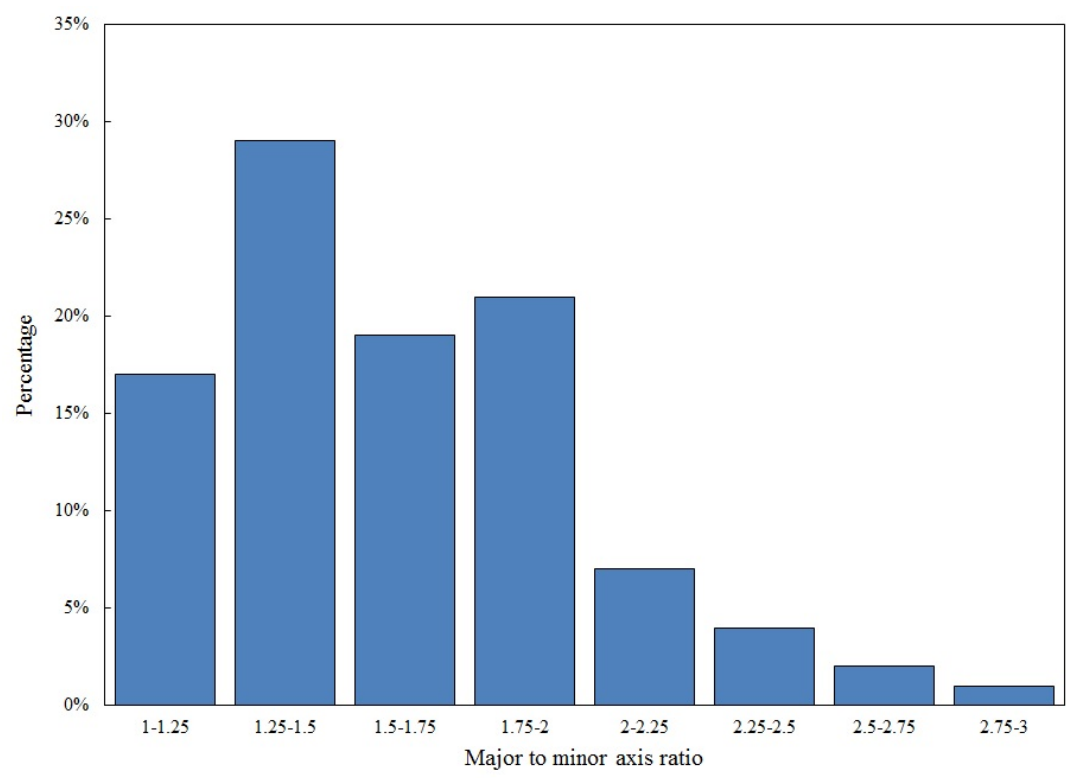

Figure B.15: Summary of the major to minor axis ratios of stretched PS.

Table B.1: Detailed characteristics of spherical and stretched PS particles.

\begin{tabular}{|c|c|c|}
\hline $\begin{array}{c}\text { Particle } \\
\text { Characteristic }\end{array}$ & $\begin{array}{c}\text { Spherical (Unstretched) PS } \\
\text { Particles }\end{array}$ & $\begin{array}{c}\text { Sretched (2:1) PS } \\
\text { Particles }\end{array}$ \\
\hline $\begin{array}{c}\text { Major axis } \\
\text { diameter }(\mathrm{nm})\end{array}$ & $208 \pm 3$ & $278 \pm 13$ \\
\hline $\begin{array}{c}\text { Minor axis } \\
\text { diameter }(\mathrm{nm})\end{array}$ & $190 \pm 3$ & $175 \pm 5$ \\
\hline Ellipticity & $0.37 \pm 0.04$ & $0.72 \pm 0.03$ \\
\hline Surface area $\left(\mu \mathrm{m}^{2}\right)$ & $0.120 \pm 0.003$ & $0.138 \pm 0.008$ \\
\hline Effective diameter & $196 \pm 0.001$ & $208 \pm 0.003$ \\
$(\mathrm{~nm})$ & & \\
\hline
\end{tabular}


The ellipticity $(e)$, surface area $(S)$, and effective diameter $\left(d_{e f f}\right)$ of the rod shape stretched particles are calculated using the following equations, where $s$ and $m$ are the radius of minor and major axis dimensions, respectively.

$$
\begin{gathered}
e=\sqrt{1-\frac{s^{2}}{m^{2}}} \\
S=2 \pi s^{2}+\frac{2 \pi s m}{e} \sin ^{-1} e \\
d_{\text {eff }}=2 \sqrt{\frac{s}{4 \pi}}
\end{gathered}
$$

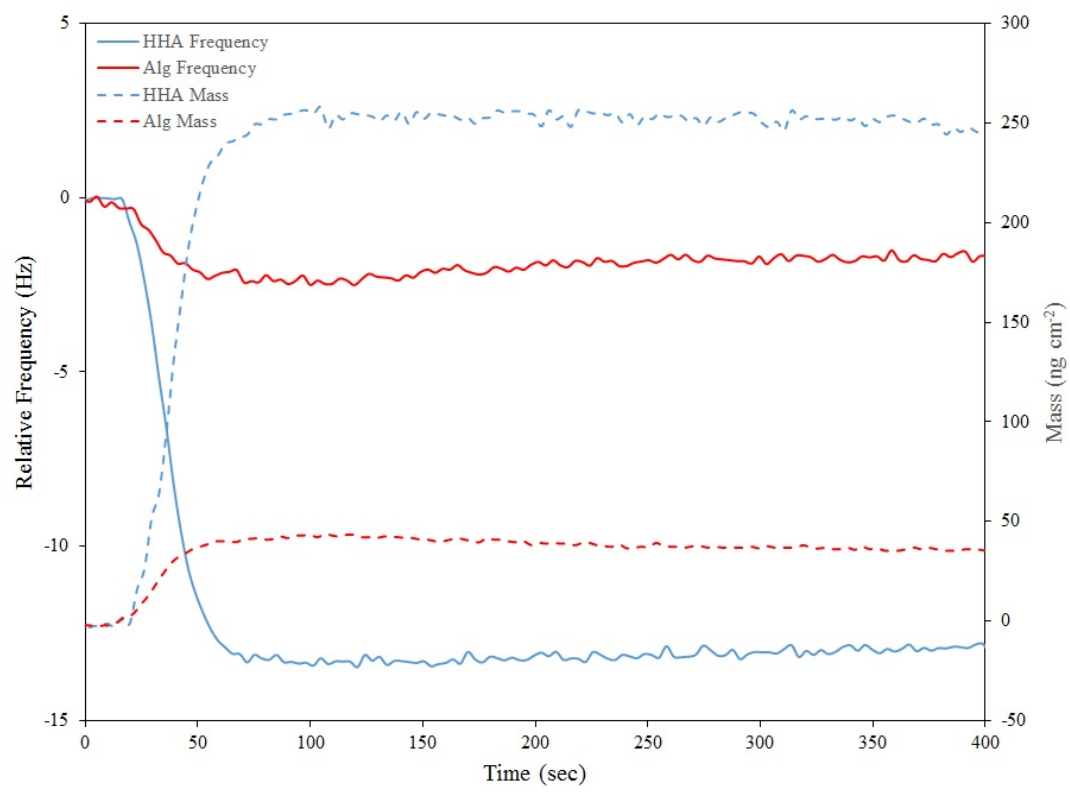

Figure B.16: The mass of HHA and alginate layers as estimated by the Voigt viscoelastic model, plotted against the relative frequency of NOM layer deposition. 


\section{Appendix C. Reynolds Number Calculation}

In order to calculate the Reynolds number (Re) of the QCM-D system, a parallel-plate channel configuration was assumed Elimelech et al. (1998).

$$
R e=\frac{\rho v L}{\mu}
$$

Eq. C.1 was used to calculate the Re of the QCM-D system where $\rho$ is the density of water, $v$ is the flow velocity within the chamber $\left(2^{*} 10^{-4} \mathrm{~ms}^{-1}\right), L$ is the half depth of the channel $\left(3.2^{*} 10^{-4} \mathrm{~m}\right)$, and $\mu$ is the viscosity of water.

\section{Appendix D. DLVO Calculation Details}

The interaction energy between the stretched nanoparticles and the collector surface was measured using surface element integration (SEI) method. SEI is a method for evaluating DLVO interaction between a particle and a flat plate Bhattacharjee and Elimelech (1997). This method accounts for the orientation dependence of the interaction energy between a flat surface and a spheroidal particle by defining the total interaction energy as follows Bhattacharjee and Elimelech (1997); Wu et al. (2013):

$$
U(h)=\int_{0}^{2 \pi} \int_{0}^{\pi} \vec{n} \cdot \vec{k}\left[E_{\text {edl }}(h)+E_{v d w}(h)\right] r^{2} \sin (\theta) d \theta d \phi
$$

${ }_{68}$ Here $\vec{n}$ is the unit outward normal to the surface element, $\vec{k}$ is the unit normal to the xy plane and directed along positive z-axis. $r, \theta$, and $\phi$ represent the radial and angular coordinates in the spherical coordinate system. $E_{\text {edl }}(h)$ and $E_{v d w}(h)$ define the electrostatic double layer and van der Waals interaction energy per unit area between two planar surfaces positioned at 
distance $h$, respectively. Stretched particles can be oriented either side on or end on. For the side on attachment between stretched particles and flat surface, the major axis position is parallel to the planar surface, however, for the end on attachment this axis is perpendicular to the collector surface plane. These two different configurations were used in the SEI method to calculate DLVO interaction energy between the stretched nanoparticles and collector surface.

The van der Waals interaction energy and electrostatic double layer interaction energy per unit area for two infinite planar surfaces separated by a distance $h$ were measured using the method by Elimelech et al. (2013) and Hogg et al. (1966), respectively:

$$
\begin{gathered}
E_{v d w}(h)=\frac{-A}{12 \pi h^{2}} \\
E_{\text {edl }}(h)=\frac{\epsilon_{0} \epsilon_{r} k}{2}\left(\varphi_{s}^{2}+\varphi_{p}^{2}\right)\left(1-\operatorname{coth}(k h)+\frac{2 \varphi_{s} \varphi_{p}}{\varphi_{s}^{2}+\varphi_{p}^{2}} \operatorname{cosech}(k h)\right) \\
k^{-1}=\left(\frac{\epsilon_{0} \epsilon_{r} K_{B} T}{2 e^{2} 1000 I_{c} N_{A}}\right)^{0.5}
\end{gathered}
$$

Here $A$ is the Hamaker constant $\left(1 \times 10^{-20} \mathrm{~J}\right), \epsilon_{0}\left(8.85 \times 10^{-12} \mathrm{C}^{2} \mathrm{~J}^{-1} \mathrm{M}^{-1}\right)$ and $\epsilon_{r}$ (80.1) are the permittivity of vacuum and relative permittivity of medium, respectively. $\varphi_{p}$ and $\varphi_{s}$ are the surface potential of ellipsoidal particle and collector surface, respectively. $k$ is the inverse Debye length, $K_{B}$ is the Boltzmann constant $\left(1.3806 \times 10^{-23} \mathrm{JK}^{-1}\right), T$ is the temperature $(293 \mathrm{~K})$, $I_{c}$ is the ionic strength of the nanoparticle suspension (M), $N_{A}$ is Avogadro's number $\left(6.02 \times 10^{23}\right)$, and $e$ is the elementary charge $\left(1.6 \times 10^{-19} \mathrm{C}\right)$. 

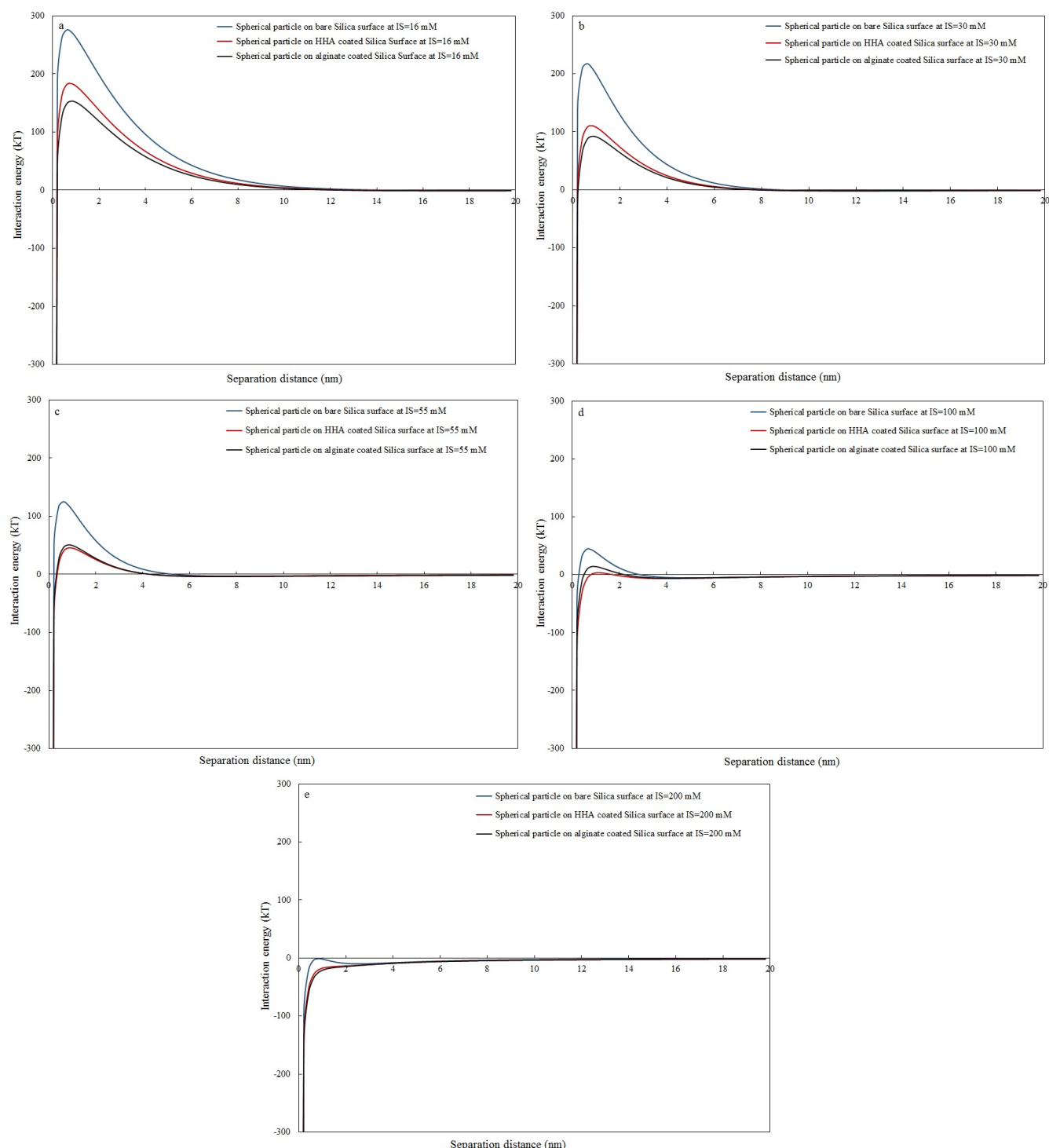

Figure D.17: Calculated DLVO interaction energy profiles between spherical PS and bare, HHA-coated, and alginate-coated silica surfaces in the presence of $16 \mathrm{mM}$ (a), $30 \mathrm{mM}$ (b), $55 \mathrm{mM}(\mathrm{c}), 100 \mathrm{mM}(\mathrm{d})$, and $200 \mathrm{mM}$ (e) NaCl. 

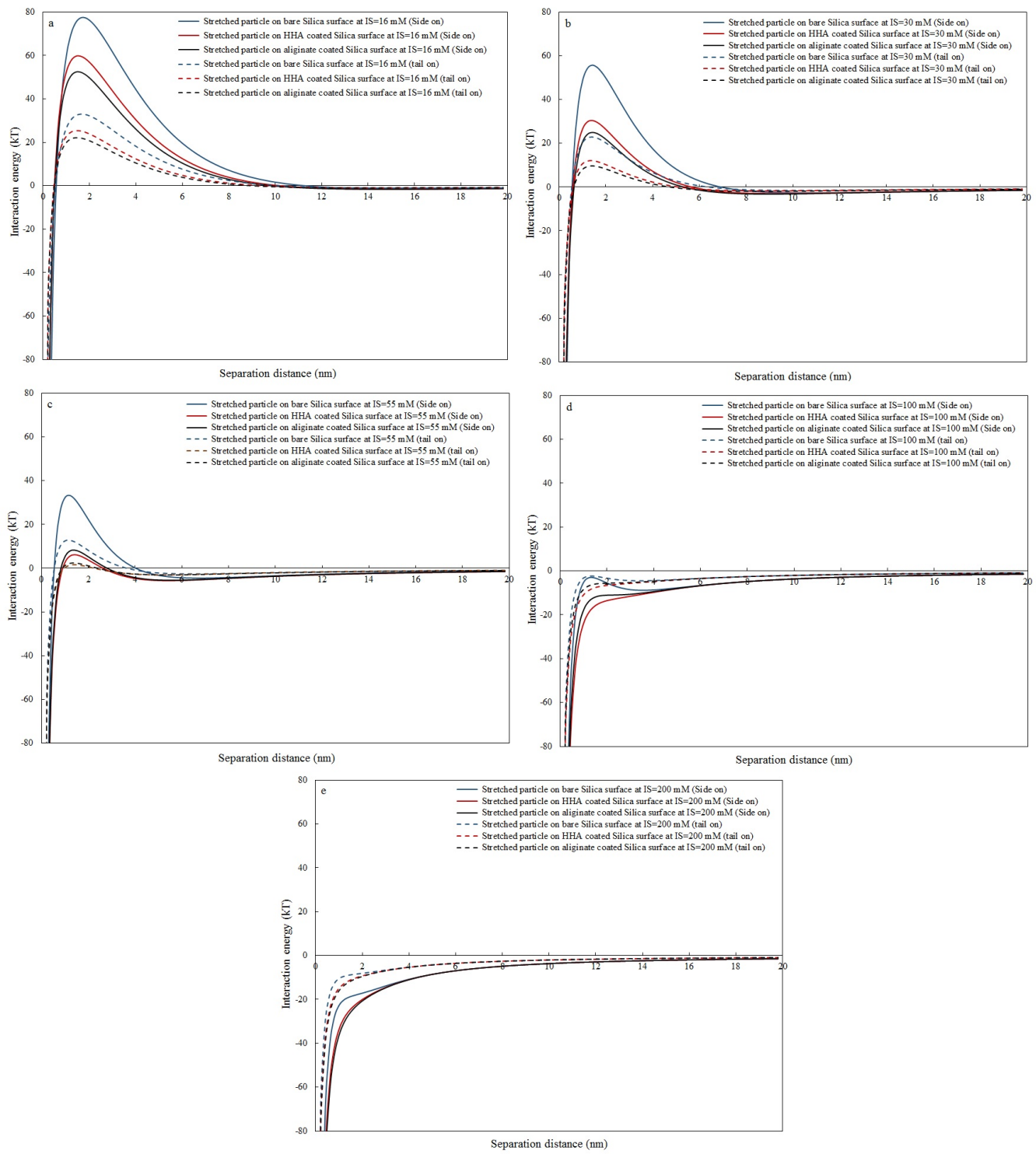

Figure D.18: Calculated DLVO interaction energy profiles between stretched PS and bare, HHA-coated, and alginate-coated silica surfaces in the presence of $16 \mathrm{mM}$ (a), $30 \mathrm{mM}$ (b), $55 \mathrm{mM}$ (c), $100 \mathrm{mM}$ (d), and $200 \mathrm{mM}$ (e) NaCl. Total interaction energy for the stretched PS was calculated as the particles approached the surface in two different orientations: major axis parallel to the surface (side on) and major axis perpendicular to the surface (end on). 